Publ. Mat. (2014), 221-253

Proceedings of New Trends in Dynamical Systems. Salou, 2012.

DOI: 10.5565/PUBLMAT_Extra14_13

\title{
THE DISCONTINUOUS MATCHING OF TWO PLANAR LINEAR FOCI CAN HAVE THREE NESTED CROSSING LIMIT CYCLES
}

\author{
Emilio Freire, Enrique Ponce, and Francisco Torres
}

\begin{abstract}
The existence and stability of limit cycles in discontinuous piecewise linear systems obtained by the aggregation of two linear systems of focus type and having only one equilibrium point is considered. By using an adequate canonical form with five parameters, a thorough study of some Poincaré maps is performed. Different bifurcations which are responsible for the appearance of crossing limit cycles are detected and parameter regions with none, one, two and three crossing limit cycles are found.

In particular, a first analytical proof of the existence, for certain differential systems in the considered family, of at least three homotopic crossing limit cycles surrounding the equilibrium point, is included. This fact has recently been numerically discovered in a particular example by S.-M. Huan and X.-S. Yang [13].
\end{abstract}

2010 Mathematics Subject Classification: 34C05, 34C07, 37G15.

Key words: Discontinuous piecewise linear systems, limit cycles, bifurcations.

\section{Introduction and preliminary results}

Piecewise linear (PWL) systems are the natural extension of linear systems in order to capture nonlinear behavior and they are extensively used in model biological processes [4] as well as electronics and mechanical devices, see for instance $[\mathbf{1}],[\mathbf{5}]$ and [18]. Even for planar cases, the qualitative analysis of different possible behavior is a rather involved task, being the detection of limit cycles an important, non-trivial problem.

The simplest family of PWL systems is the planar case constituted by two linear systems matched along a straight line. The case of planar PWL systems with continuous matching along the straight line was carefully studied in $[\mathbf{8}]$, where some canonical forms with only four parameters were obtained. In particular, by using these canonical forms,

This work was partially supported by a MINECO/FEDER grant number MTM200907849 and the grant EXC/P08-FQM-03770 of Junta de Andalucía. 
the existence at most of one isolated invariant closed curve (a limit cycle or an isolated homoclinic loop) was shown.

Always within the planar case, the discontinuous matching of two linear systems has recently deserved the attention by researchers. After the Filippov extension of the concept of solution to the discontinuity line [7], many works dealing with discontinuous PWL systems have appeared. In $[\mathbf{1 0}]$ and $[\mathbf{1 1}]$, PWL systems with $\mathbb{Z}_{2}$ symmetry were investigated. In [17] particular Filippov systems were both linear dynamics are of saddle type were studied. The Hopf bifurcation in non-smooth systems was studied in [12], $[\mathbf{3}]$ and $[\mathbf{1 9}]$. In fact, in $[\mathbf{1 2}]$ authors conjectured that the maximum number of limit cycles for the class of systems under study is exactly two. However, in $[\mathbf{1 3}]$ it was numerically obtained the existence of three limit cycles for a planar PWL system, but no analytical proof was provided. Recently, a computer assisted proof has been available, see [15], and based on such a proof it is shown in [2] that the three limit cycles can be obtained through a boundary focus bifurcation. In this paper, as one of the goals to be achieved, we will give the lacking analytical proof. Even we conjecture that three is indeed the maximum number of limit cycles to be found, it is still needed a proof of this fact.

As claimed in [13], the study of discontinuous PWL systems is a difficult task because of the absence of a canonical form able to cope with a sufficiently broad class of systems. To fill this gap, in [9] a Liénard-like canonical form with seven parameters was obtained; under the assumption of both linear focus dynamics, a simpler canonical form with only five parameters was also achieved. Even for the two foci cases, the number of qualitatively different phase portraits is great, so the study made in [9] was restricted to the case without equilibria in both open half planes. Particular instances of this last situation previously appeared in $[\mathbf{1 6}]$.

This paper can be properly considered as a continuation of the work in [9]. Thus, we consider PWL systems where both dynamics are of focus type and the system has only one real equilibrium, procrastinating the cases with two real equilibria for a future work. We investigate the dynamical behavior of this family by studying mainly the existence and stability of the so called crossing limit cycles. We will provide, following a bifurcational approach, a particular mechanism to explain the existence of three limit cycles in a certain open set of the parameter space. Thus, we confirm analytically the phenomenon numerically observed in $[\mathbf{1 3}]$ for a specific system. Furthermore, we show that the non-genericity of the example in $[\mathbf{1 3}]$, where the two involved vector fields vanish at the same point, is not needed at all. 
In the study of planar piecewise linear systems with two zones separated by a straight line, we can assume without loss of generality that the discontinuity line is $\Sigma=\{(x, y): x=0\}$. If we define now the open sets

$$
S^{-}=\{(x, y): x<0\}, \quad S^{+}=\{(x, y): x>0\},
$$

the systems to be studied can be written as

(1) $\dot{\mathbf{x}}=\mathbf{F}(\mathbf{x})= \begin{cases}\mathbf{F}^{+}(\mathbf{x})=\left(F_{1}^{+}(\mathbf{x}), F_{2}^{+}(\mathbf{x})\right)^{T}=A^{+} \mathbf{x}+\mathbf{b}^{+}, & \text {if } \mathbf{x} \in S^{+}, \\ \mathbf{F}^{-}(\mathbf{x})=\left(F_{1}^{-}(\mathbf{x}), F_{2}^{-}(\mathbf{x})\right)^{T}=A^{-} \mathbf{x}+\mathbf{b}^{-}, & \text {if } \mathbf{x} \in S^{-} .\end{cases}$

Here $\mathbf{x}=(x, y)^{T} \in \mathbb{R}^{2}, A^{+}=\left(a_{i j}^{+}\right)$and $A^{-}=\left(a_{i j}^{-}\right)$are $2 \times 2$ constant matrices and $\mathbf{b}^{+}=\left(b_{1}^{+}, b_{2}^{+}\right)^{T}, \mathbf{b}^{-}=\left(b_{1}^{-}, b_{2}^{-}\right)^{T}$ are constant vectors of $\mathbb{R}^{2}$.

The restriction of the system to each half-plane is a linear system and so, orbits are well defined while they evolve without touching the $y$-axis. However, we must adopt some criterion to define the orbits arriving at the discontinuity line. When a solution arrives at the point $(0, y)$ belonging to $\Sigma$, different things can occur.

If $F_{1}^{+}(0, y) F_{1}^{-}(0, y)>0$ then both vector fields are transversal to the discontinuity line and their normal components have the same sign. In this case, we will assume that orbits are concatenated in the natural way. We say that this point is a crossing point, so that the $\operatorname{crossing}$ set $\Sigma^{c}$ is defined as follows

$$
\Sigma^{c}=\left\{(0, y): F_{1}^{+}(0, y) F_{1}^{-}(0, y)=\left(a_{12}^{+} y+b_{1}^{+}\right)\left(a_{12}^{-} y+b_{1}^{-}\right)>0\right\} .
$$

If $F_{1}^{+}(0, y) F_{1}^{-}(0, y) \leqslant 0$ then we say that this point is a sliding point. The sliding set $\Sigma^{s}$, which is the complementary set in $\Sigma$ of the crossing set, is defined as,

$$
\Sigma^{s}=\left\{(0, y): F_{1}^{+}(0, y) F_{1}^{-}(0, y)=\left(a_{12}^{+} y+b_{1}^{+}\right)\left(a_{12}^{-} y+b_{1}^{-}\right) \leqslant 0\right\} .
$$

As usual, when a orbit arrives at the sliding set we will adopt the Filippov convex method, see [14].

Since we are interested in the study of non-trivial periodic orbits, we look for posible periodic orbits not totally contained in either $S^{+}$or $S^{-}$. If these orbits have sliding points they will be called sliding periodic orbits. Otherwise, we speak of crossing periodic orbits, whose study is the main goal of this paper.

When $a_{12}^{+} a_{12}^{-} \leqslant 0$, it is easy to see from (2) that the crossing set, if it exists, is an open interval of the $y$-axis, unbounded for $a_{12}^{+} a_{12}^{-}=0$ and bounded for $a_{12}^{+} a_{12}^{-}<0$. In any case, the $x$-component of both vector fields has constant sign at the crossing set and so elementary qualitative arguments preclude the existence of crossing periodic orbits. This case 
will not be considered in the rest of the paper and we assume in the sequel $a_{12}^{+} a_{12}^{-}>0$.

As it was shown in Proposition 3.1 of $[\mathbf{9}]$, discontinuous planar piecewise linear systems (1) under the assumption $a_{12}^{+} a_{12}^{-}>0$ can be transformed by means of a certain homeomorphism into the Liénard canonical form,

$$
\begin{aligned}
& \dot{\mathbf{x}}=\left(\begin{array}{cc}
T^{-} & -1 \\
D^{-} & 0
\end{array}\right) \mathbf{x}-\left(\begin{array}{c}
0 \\
a^{-}
\end{array}\right) \quad \text { if } \mathbf{x} \in S^{-}, \\
& \dot{\mathbf{x}}=\left(\begin{array}{cc}
T^{+} & -1 \\
D^{+} & 0
\end{array}\right) \mathbf{x}-\left(\begin{array}{c}
-b \\
a^{+}
\end{array}\right) \quad \text { if } \mathbf{x} \in S^{+},
\end{aligned}
$$

where $T^{+}, T^{-}$denote the traces and $D^{+}, D^{-}$denote the determinants of matrices $A^{+}$and $A^{-}$, respectively.

It is worth noting that the discontinuity line remains invariant under the above homeomorphism. Moreover, crossing and sliding sets, tangency points and boundary equilibria of the original system are transformed by the quoted homeomorphism into sets and points of the same type for system (4). More precisely, such a homeomorphism induces a topological equivalence between systems (1) and (4) for all their orbits not intersecting the sliding set or for the ones having at most the tangency points in common with the sliding set. Also, the attractive or repulsive character of the sliding set is preserved, see $[\mathbf{9}]$.

As mentioned before, we assume that both dynamics are of focus type, that is $T^{2}-4 D<0$ in the two zones. Then, we know that matrices $A^{ \pm}$ have the eigenvalues $\lambda^{ \pm}=\alpha^{ \pm} \pm i \omega^{ \pm}$with $\omega^{ \pm}>0$, so that in the canonical form (4) we have $T^{ \pm}=2 \alpha^{ \pm}$and $D^{ \pm}=\left(\alpha^{ \pm}\right)^{2}+\left(\omega^{ \pm}\right)^{2}$.

By introducing the parameters

$$
\gamma_{R}=\frac{\alpha^{+}}{\omega^{+}}, \quad \gamma_{L}=\frac{\alpha^{-}}{\omega^{-}}, \quad a_{R}=\frac{a^{+}}{\omega^{+}}, \quad a_{L}=\frac{a^{-}}{\omega^{-}},
$$

and doing the change of variables (different for each half-plane)

$$
(x, y, t) \rightarrow\left(\frac{x}{\omega(x)}, y, \frac{t}{\omega(x)},\right), \text { where } \omega(x)= \begin{cases}\omega^{-} & \text {if } x<0 \\ \omega^{+} & \text {if } x>0\end{cases}
$$

it was proved in Proposition 4.1 of $[\mathbf{9}]$ that canonical form (4) reduces to

$$
\begin{aligned}
& \dot{\mathbf{x}}=\left(\begin{array}{cc}
2 \gamma_{L} & -1 \\
1+\gamma_{L}^{2} & 0
\end{array}\right) \mathbf{x}-\left(\begin{array}{c}
0 \\
a_{L}
\end{array}\right) \quad \text { if } \mathbf{x} \in S^{-}, \\
& \dot{\mathbf{x}}=\left(\begin{array}{cc}
2 \gamma_{R} & -1 \\
1+\gamma_{R}^{2} & 0
\end{array}\right) \mathbf{x}-\left(\begin{array}{c}
-b \\
a_{R}
\end{array}\right) \quad \text { if } \mathbf{x} \in S^{+},
\end{aligned}
$$


where for the sake of convenience we will put

$$
a_{L}=\left(1+\gamma_{L}^{2}\right) x_{L}, \quad a_{R}=\left(1+\gamma_{R}^{2}\right) x_{R} .
$$

The canonical form (5) is invariant under the transformations shown in Table 1 so that, after applying if needed symmetries $\Pi_{1}$ or $\Pi_{2}$, it is not restrictive to assume $b \geqslant 0$.

\begin{tabular}{|c|c|c|}
\hline$\Pi_{1}$ & $\Pi_{2}$ & $\Pi_{3}$ \\
\hline$x \rightarrow x$ & $x \rightarrow-x$ & $x \rightarrow-x$ \\
\hline$y \rightarrow-y$ & $y \rightarrow y+b$ & $y \rightarrow-y-b$ \\
\hline$t \rightarrow-t$ & $t \rightarrow-t$ & $t \rightarrow t$ \\
\hline$\gamma_{L} \rightarrow-\gamma_{L}$ & $\gamma_{L} \rightarrow-\gamma_{R}$ & $\gamma_{L} \rightarrow \gamma_{R}$ \\
\hline$\gamma_{R} \rightarrow-\gamma_{R}$ & $\gamma_{R} \rightarrow-\gamma_{L}$ & $\gamma_{R} \rightarrow \gamma_{L}$ \\
\hline$x_{L} \rightarrow x_{L}$ & $x_{L} \rightarrow-x_{R}$ & $x_{L} \rightarrow-x_{R}$ \\
\hline$b \rightarrow-b$ & $b \rightarrow-b$ & $b \rightarrow b$ \\
\hline$x_{R} \rightarrow x_{R}$ & $x_{R} \rightarrow-x_{L}$ & $x_{R} \rightarrow-x_{L}$ \\
\hline
\end{tabular}

TABLe 1. Symmetries of canonical form (5).

When $b>0$, the sliding set of our canonical form is given by

$$
\Sigma^{s}=\{(0, y): 0 \leqslant y \leqslant b\},
$$

shrinking to the origin for $b=0$. Accordingly, the crossing set is the open set $\Sigma^{c}=\Sigma \backslash \Sigma^{s}$. In the case $b>0$, the convex method of Filippov leads to the sliding vector field,

$$
\dot{x}=0, \quad \dot{y}=g(y)=\frac{a_{L}-a_{R}}{b} y-a_{L}, \quad 0 \leqslant y \leqslant b,
$$

to be considered only on the sliding set.

Note that from [14], as the $x$-components of both vector fields at the sliding set $\Sigma^{s}$ points outwards from $\Sigma^{s}$, the sliding set is unstable in the normal direction, so that the sliding set is usually called escaping sliding set.

For the points where one of the right hand-sides in (5) vanishes, we distinguish as usual between real and virtual equilibrium points. More precisely, $\mathbf{e}_{L}=\left(x_{L}, 2 \gamma_{L} x_{L}\right)$ is a real equilibrium point when $x_{L} \leqslant 0$, being virtual when $x_{L}>0$. In the critical case $x_{L}=0$ we speak of a 
boundary equilibrium point. Note that the origin is a visible tangency point for $x_{L}<0$ (invisible, for $x_{L}>0$ ) for the left vector field, see [14].

Similarly, $\mathbf{e}_{R}=\left(x_{R}, 2 \gamma_{R} x_{R}\right)$ is a real equilibrium when $x_{R} \geqslant 0$ (boundary equilibrium point if $x_{R}=0$ ) and virtual when $x_{R}<0$. Moreover, the right vector field has a visible tangency point for $x_{R}>0$ (invisible, for $x_{R}<0$ ) at the point $(0, b)$. Note that for $b=0$ the origin is a double tangency point, provided that $x_{L} x_{R} \neq 0$.

Points $(0, y) \in \Sigma^{s}$ with $g(y)=0$ in (7) act as well in some sense as equilibria of system (1) and they are called pseudo-equilibria for system (5). From (7) we deduce that our system has a pseudo-equilibrium point only when $a_{R} a_{L}<0$, and then it is given by $\left(0, \bar{y}_{p}\right)$ with

$$
\bar{y}_{p}=\frac{a_{L} b}{a_{L}-a_{R}} .
$$

Note that at the pseudo-equilibrium point $\left(0, \bar{y}_{p}\right)$ the two vector fields of (5) become anticollinear.

Next, in Section 2 our main results are presented.

\section{Statement of main results}

We concentrate our attention in system (5) with $b \geqslant 0$ assuming that there exists only one equilibrium point in the interior of the zones $S^{-}, S^{+}$, so that we must have $x_{R} x_{L}>0$. The case when the system has no real equilibrium points in the interior of the open regions $S^{-}, S^{+}$was studied in $[\mathbf{9}]$, where it was shown the possible existence of two crossing limit cycles.

Without loss of generality, we can assume $x_{R}<0$ and $x_{L}<0$, that is, the only equilibrium is located in the left zone, otherwise it suffices to apply the transformation $\Pi_{3}$ of Table 1 .

Next, we give our first result on limit cycles which is related to the case when the sliding set reduces to the origin.

Theorem 1 (singleton sliding set, $b=0$ ). Assuming in system (5) the conditions $b=0, x_{R}<0$ and $x_{L}<0$, the following statements hold.

(a) If $\gamma_{R} \gamma_{L} \geqslant 0$ and $\gamma_{R}+\gamma_{L} \neq 0$, then there are no crossing periodic orbits.

(b) If $\gamma_{R}=\gamma_{L}=0$, then every orbit is a periodic orbit and the configuration is a global center.

(c) If $\gamma_{R} \gamma_{L}<0$ and $\gamma_{L}\left(\gamma_{R}+\gamma_{L}\right) \geqslant 0$, then there are no crossing periodic orbits.

(d) If $\gamma_{R} \gamma_{L}<0$ and $\gamma_{L}\left(\gamma_{R}+\gamma_{L}\right)<0$, then there is only one crossing periodic orbit which is stable for $\gamma_{L}>0$ and unstable for $\gamma_{L}<0$. 
Since the equilibrium point is in the interior of the left zone, its stability is clearly determined by the sign of parameter $\gamma_{L}$. Later on, we will establish that the stability of the point at infinity, that is the equator of the corresponding Poincaré sphere, is determined by the sign of $\gamma_{L}+\gamma_{R}$. Hence, roughly speaking, Theorem 1 states that we have one limit cycle on the condition that the origin and the infinity have different stabilities.

For $b \neq 0$, the sliding set is a segment with two tangency points at its endpoints: a visible tangency at the origin for the left vector field and a invisible tangency at the point $(0, b)$ for the right vector field. However, this sliding segment is stable for $b<0$ and unstable for $b>0$. Such a change of stability is in fact the bifurcation phenomenon called $V I_{1}$ in $[\mathbf{1 4}]$.

When $b>0$, the sliding vector field (7) for $x_{L}<0$ and $x_{R}<0$ must be taken into account. Then we have $\dot{y}>0$ in the sliding dynamics and there are no pseudo-equilibrium points. Since the sliding set is repulsive, the only orbit arriving at the sliding set is the tangent one while for reversing time all the sliding orbits leave the sliding set at the origin. Then, in order to establish the existence of sliding periodic orbits we only have to study the orbits through the origin. According to $[\mathbf{1 4}]$, note that it is possible the existence of invariant closed curves backwards in time which are not invariant forward in time; in this case, we speak of backward-invariant closed curves.

Next, let us consider the existence of sliding periodic orbits forward and backward in time.

In forward time, the tangent orbit at the origin enters in the sliding set $\Sigma^{s}$ and slides along $\Sigma^{s}$ towards the point $(0, b)$. Next, it evolves in the left zone around the equilibrium point $\mathbf{e}_{L}$, either finally arriving at $\mathbf{e}_{L}$ or coming back to the line $x=0$ at a point $\left(0, y_{1}\right)$ with $y_{1} \leqslant 0$. If $y_{1}<0$, then from the point $\left(0, y_{1}\right)$, the orbit never returns to the sliding set, so that we have no sliding periodic orbits. If $y_{1}=0$, we have a sliding periodic orbit. Thus, the only sliding periodic orbit in forward time appears when the orbit starting at the point $(0, b)$ passes through the origin.

To analyze the possible sliding periodic orbits backward in time, we can discard the case $\gamma_{L}>0$, for then when an orbit abandon the sliding set at the origin never returns to the sliding set, approaching the equilibrium point $\mathbf{e}_{L}$. Thus, assume to fix ideas that $\gamma_{L} \leqslant 0$ and let us introduce the point $\hat{\mathbf{p}}=(0, \hat{y})$ with $\hat{y} \geqslant 0$ such that the orbit forwards in time of the left vector field starting at the point $\hat{\mathbf{p}}$ passes through the origin. Also, we define the point $\mathbf{p}^{*}=\left(0, y^{*}\right)$ with $y^{*}<b$ such that the orbit forward in time of the right vector field starting at the point $\mathbf{p}^{*}$ 
passes through the point $\hat{\mathbf{p}}$. Different cases appear by considering the position of the points $\hat{\mathbf{p}}$ and $\mathbf{p}^{*}$.

If $0 \leqslant \hat{y} \leqslant b$ then the point $\hat{\mathbf{p}}$ belong to the sliding set. The case $\hat{y}=0$ corresponds with a linear center in the left zone, that is $\gamma_{L}=0$, and we trivially see that the system has no sliding periodic orbits. Assume $\hat{y}>0$, then, always backward in time, the orbit slides along the line $x=$ 0 to the origin. Consequently, we detect by going backward in time a closed sliding orbit which only uses the left zone and surrounds the equilibrium $\mathbf{e}_{L}$, see Figure 1 . Note that if $\hat{y}=b$, then the limit cycle uses the full sliding set and thus we have the only sliding periodic orbit that can also be detected forward in time.

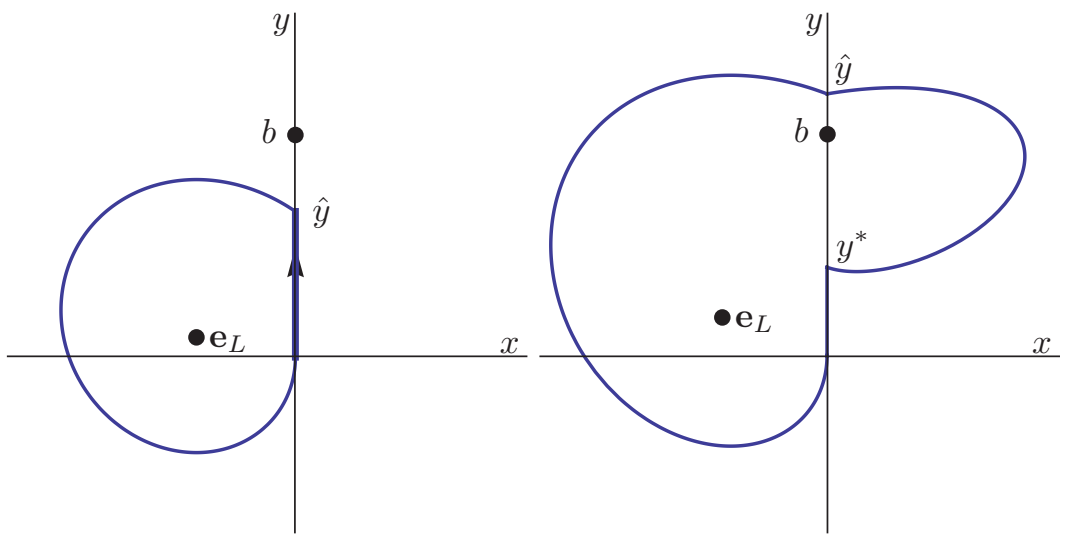

Figure 1. Left panel: The sliding orbit lives in only one zone when $\hat{y} \leqslant b$. Right panel: Sliding orbit living in the two zones for when $\hat{y}>b$. The arrow indicates the sense for the forward time.

If $\hat{y}>b$, then the point $\hat{\mathbf{p}}$ belongs to the crossing set. Next, by going backward in time, the orbit through the point $\hat{\mathbf{p}}$ will enter in the region $S^{+}$surrounding the tangency point $(0, b)$ to come back again to the line $x=0$ at a point $\left(0, y^{*}\right)$ with $y^{*}<b$, see Figure 1 . If $y^{*} \geqslant 0$, then the orbit slides back to the origin forming a unstable sliding limit cycle that uses both regions $S_{L}$ and $S_{R}$. If $y^{*}<0$, then the backward orbits starting at the sliding set never returns to it and there are no sliding limit cycles.

Remark 1 . In the particular case where $\hat{y}>b$ and the orbit starting at the origin arrives backward in time again to the origin, that is $y^{*}=0$, 
we have a backward-invariant closed curve which only shares the origin with the sliding set, see Figure 2. This orbit is called crossing critical cycle, see Section 4.1.4 in [14], and represents the boundary between the sliding periodic orbits and the crossing periodic orbits.

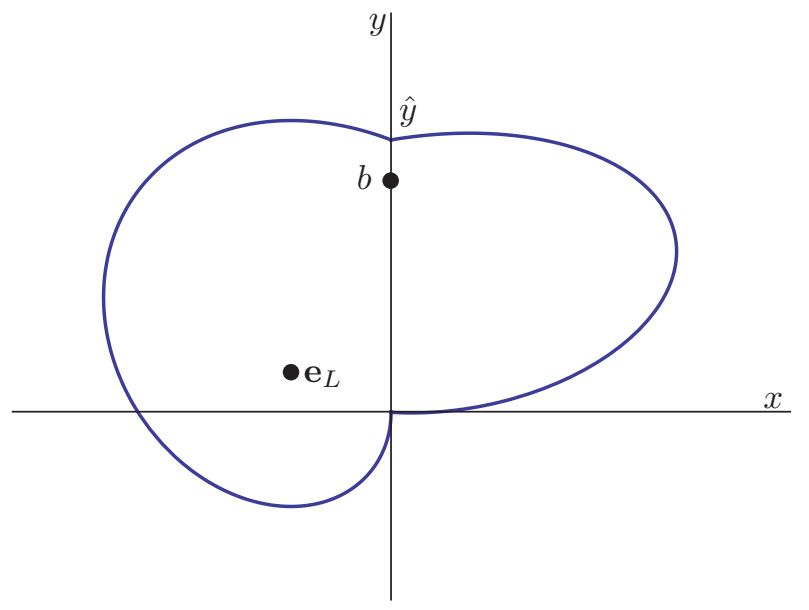

Figure 2. The crossing critical cycle described in Remark 1.

The above discussion shows that if there exists a sliding periodic orbit for system (5), then it is unique. In fact we can state the following proposition.

Proposition 1 (sliding periodic orbits). Assume that $x_{L}<0, x_{R}<0$, $b>0$ in system (5). When $\gamma_{L} \geqslant 0$ the system has no sliding periodic orbits; if $\gamma_{L}<0$ and regarding the values $\hat{y}>0$ and $y^{*}<b$ above defined, the following statements hold.

(a) If $\hat{y}<b$ then there is one sliding orbit backward in time which lives in the left zone and it is unstable.

(b) If $\hat{y}=b$ then there is one sliding orbit both backward and forward in time, which lives in the left zone and it is unstable.

(c) If $\hat{y}>b$ then the following cases arise.

(i) If $0<y^{*}<b$ then there is one sliding periodic orbit backward in time which lives in the two zones and it is unstable.

(ii) If $y^{*}=0$ then there is one unstable crossing critical cycle which is unstable.

(iii) If $y^{*}<0$ then there are no sliding periodic orbit. 
In the next theorems we study the number of crossing periodic orbits. We need to introduce first a critical value of the parameter $b$, which corresponds to the existence of the crossing critical cycle when $\gamma_{L}<0$.

Proposition 2. Assume that $x_{L}<0, x_{R}<0$ and $\gamma_{L}<0$ in system (5). Then there exists one smooth function $b=b_{C C}\left(\gamma_{R}\right)$ with $0<b_{C C}\left(\gamma_{R}\right)<$ $\hat{y}$ and $b_{C C}(0)=\hat{y} / 2$, defined for every value of $\gamma_{R}$ such that for $b=$ $b_{C C}\left(\gamma_{R}\right)$ the system has one unstable crossing critical cycle. In addition there exists $\varepsilon>0$ such that for $b_{C C}\left(\gamma_{R}\right)-\varepsilon<b<b_{C C}\left(\gamma_{R}\right)$ there exists one unstable crossing periodic orbit which bifurcates from the crossing critical cycle.

Before proceeding further, it should be noticed the detection for these planar Filippov piecewise linear systems of two bifurcations reported in the literature. Considering Proposition 1, in passing from the situations described in statements (a) to (b) and then to (c)(i), we see that from the existence of a one-zonal sliding periodic orbit we arrive at one two-zonal sliding periodic orbit through a critical crossing cycle, that is, we detect the so called buckling bifurcation in [14], also named switching-sliding bifurcation in $[\mathbf{6}]$, see Figure 1. Also, by considering Proposition 2 we see that by decreasing the value of parameter $b$, we pass from a critical crossing cycle to a one unstable crossing periodic orbit and we get the so called $C C$ bifurcation in [14], also named crossing-sliding bifurcation in $[6]$.

Now we give a lower bound for the maximum number of crossing periodic orbits, disregarding for the sake of brevity not only the possible existing sliding periodic orbits but also the crossing critical cycle that exists for $b=b_{C C}$, see Proposition 2. We split the study in two different situations, speaking of opposite divergences when $\gamma_{L} \gamma_{R}<0$ and nonopposite divergence otherwise. For this last case, we can give the exact number of crossing periodic orbits for all parameter values.

Theorem 2 (non-opposite divergences). Assume that $x_{L}<0, x_{R}<0$, $b>0$, and $\gamma_{L} \gamma_{R} \geqslant 0$ in system (5), then the following cases arise.

(a) If $\gamma_{L} \geqslant 0$ and $\gamma_{R} \geqslant 0$, then there are no crossing periodic orbits.

(b) If $\gamma_{L}=0$ and $\gamma_{R}<0$, then there is one stable crossing periodic orbit. This limit cycle surrounds a bounded periodic annulus living in the left zone and tangent to the origin.

(c) If $\gamma_{L}<0$ and $\gamma_{R} \leqslant 0$, then there exists a value $b_{S N}$ with $0<b_{S N}<$ $b_{C C}$ such that there are no crossing periodic orbits for $b<b_{S N}$, two crossing periodic orbits with opposite stabilities for $b_{S N}<b<$ 
$b_{C C}$, and only one stable crossing periodic orbit for $b \geqslant b_{C C}$. For $b=b_{S N}$ the system has only one crossing periodic orbit, which is semi-stable.

The possible cases of opposite divergences are much more involved, and we must distinguish whether the equilibrium point is stable or not. In the case of unstable equilibrium we can characterize completely the number of crossing periodic orbits.

Theorem 3 (unstable equilibrium). Assume that $x_{L}<0, x_{R}<0$, $b>0, \gamma_{L}>0$ and $\gamma_{R}<0$ in system (5), then the following statements hold.

(a) If $\gamma_{L}+\gamma_{R} \geqslant 0$, then there are no crossing periodic orbits.

(b) For $\gamma_{L}+\gamma_{R}<0$ there is only one crossing periodic orbit which is stable.

In Theorems 1 and 3, we have detected a change in the number of crossing limit cycles depending on the sign of the value $\gamma_{L}+\gamma_{R}$. Obviously, this is related to the stability of the periodic orbit at infinity which exists due to the focus dynamics in both half-planes. We must expect a Hopf bifurcation at infinity when $\gamma_{L}+\gamma_{R}=0$. As we will see in Theorem $4(\mathrm{e})$, the character (supercritical or subcritical) changes at the value $b_{\infty}=2\left(x_{L}+x_{R}\right) \gamma_{L}$, of the parameter $b$, and so the point $\left(-\gamma_{L}, b_{\infty}\right)$ plays a special role on the plane $\left(\gamma_{R}, b\right)$. On the other hand, when the equilibrium is stable, we can give information about the number of crossing periodic orbits only in certain regions of the parameter space.

Theorem 4 (stable equilibrium and extremal values of $b$ ). Assuming $x_{L}<0, x_{R}<0, \gamma_{L}<0$ and $\gamma_{R}>0$ in system (5) the following statements hold.

(a) If $\gamma_{L}+\gamma_{R}<0$ and $b \geqslant b_{C C}$, then there is at least one stable crossing periodic orbit.

(b) If $\gamma_{L}+\gamma_{R} \leqslant 0$ and $b<2 x_{L} \gamma_{L}$, then there are no crossing periodic orbits.

(c) If $\gamma_{L}+\gamma_{R}>0$ and $b<b_{C C}$, then there is at least one unstable crossing periodic orbit.

(d) If $\gamma_{L}+\gamma_{R} \geqslant 0$, then there exists a constant $M>0$ such that for all $b>M$ there are no crossing periodic orbits.

(e) If $b<b_{\infty}$, then there exists $\varepsilon_{1}>0$ such that for $-\gamma_{L}<\gamma_{R}<\gamma_{L}+$ $\varepsilon_{1}$, there is at least one unstable crossing periodic orbit and when $b>b_{\infty}$, then there exists $\varepsilon_{2}>0$ such that for $-\varepsilon_{2}-\gamma_{L}<\gamma_{R}<\gamma_{L}$, there is at least one stable crossing periodic orbit. 
Next, as the most interesting situation, we study some cases when the parameter $b$ belongs to a neighborhood of $b_{C C}\left(\gamma_{R}\right)$, see Proposition 2 .

Theorem 5 (stable equilibrium, $b$ near $b_{C C}$ ). Assuming that $x_{L}<0$, $x_{R}<0, \gamma_{L}<0$ and $\gamma_{R}>0$ in system (5), the following statements hold.

(a) If $\gamma_{L}+\gamma_{R}<0$ there exists $\varepsilon>0$ such that for $b_{C C}-\varepsilon<b<b_{C C}$ the system has at least two crossing periodic orbits with opposite stabilities.

(b) Provided that $\hat{y}<b_{\infty}$, the following statements also hold.

(i) Assume $\gamma_{R}=-\gamma_{L}$. Then, there exists $\varepsilon_{0}>0$ such that for $b_{C C} \leqslant b<b_{C C}+\varepsilon_{0}$ the system has at least a stable crossing periodic orbit. In addition, there exists $\varepsilon_{1}>0$ such that for $b_{C C}-\varepsilon_{1}<b<b_{C C}$ the system has at least two crossing periodic orbits with opposite stabilities.

(ii) There exists $\varepsilon_{2}>0$ such that for $-\gamma_{L}<\gamma_{R}<-\gamma_{L}+\varepsilon_{2}$ and $b=b_{C C}\left(\gamma_{R}\right)$ the system has at least two crossing periodic orbits with opposite stabilities. Furthermore, for $-\gamma_{L}<\gamma_{R}<$ $-\gamma_{L}+\varepsilon_{2}$ there exists $\varepsilon_{3}\left(\gamma_{R}\right)>0$ such that for $b=b_{C C}\left(\gamma_{R}\right)-$ $\varepsilon_{3}\left(\gamma_{R}\right)$ the system has at least three nested crossing periodic orbits being stable the intermediate one and unstable the two other.

We emphasize that with the proof of statement (b)(ii) we have obtained for the first time an analytical argument to show the existence of at least three crossing limit cycles in two-zonal piecewise linear discontinuous systems, for which only a computer assisted proof was available, see $[\mathbf{1 5}]$. As mentioned in the introduction, this situation was first detected in a rather specific example appeared in $[\mathbf{1 3}]$.

To illustrate the assertions given in Theorems 1 to 5, in Figures 3 and 4 , we show a partial representative bifurcation set in the plane $\left(\gamma_{R}, b\right)$. To fix the value of $\gamma_{L}$, we have chosen $x_{L}=-1$ and imposed a time $t_{L}=$ $3 \pi / 2$ for the orbit coming from the point $(0, \hat{y})$ to arrive at the origin. We obtained $\gamma_{L} \approx-0.27441$ and $\hat{y} \approx 3.91858$. After fixing $x_{R}=-7$, we are under the hypotheses of statement (b) of Theorem 5 , since $b_{\infty}=$ $2\left(x_{L}+x_{R}\right) \gamma_{L} \approx 4.39057>\hat{y}$.

In Figure 3 and in the magnified version of Figure 4, we draw for the above values the vertical line $\gamma_{R}=-\gamma_{L}$ in green. By using a standard continuation code, applied to the equations for periodic orbits obtained from the linear flow in each zone, we also draw three bifurcation curves: in red, the graph of the curve $b=b_{C C}\left(\gamma_{R}\right)$, recall Proposition 2; in blue, the predicted curve $b_{S N}$ in Theorem 2 for $\gamma_{R} \leq 0$ which persists 
for $\gamma_{R}>0$; also in blue, we have numerically detected another saddlenode bifurcation curve of crossing limit cycles $b_{S N I}$, emanating from the point $\left(-\gamma_{L}, b_{\infty}\right)$.

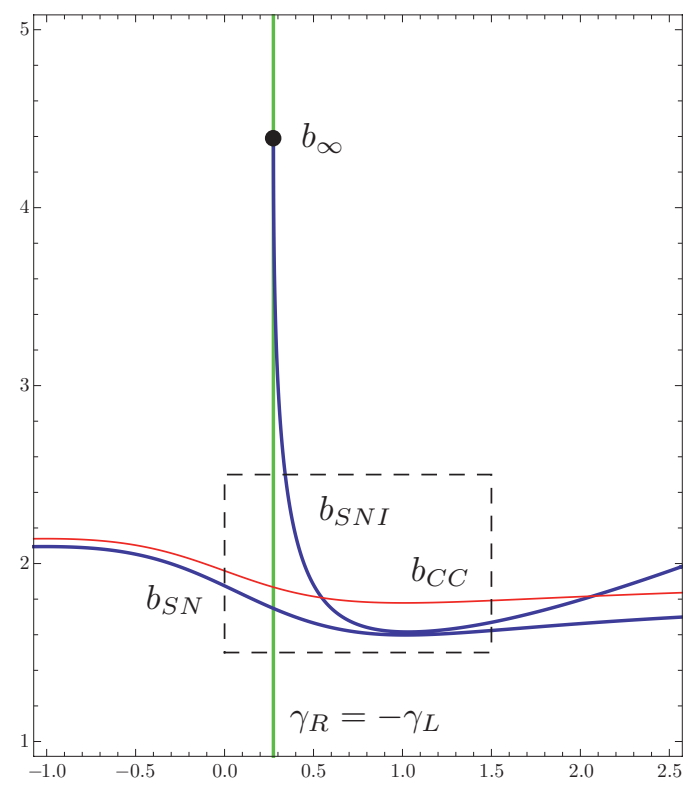

Figure 3 . The curves $b_{C C}\left(\gamma_{R}\right), b_{S N}\left(\gamma_{R}\right)$ and $\gamma_{R}=-\gamma_{L}$ for the values $x_{L}=-1, x_{R}=-7$ and $\gamma_{L} \approx-0.27441$, in the plane $\left(\gamma_{R}, b\right)$. The dashed box is zoomed in Figure 4 .

At the line $b=0$, not shown in Figure 3, the sliding set becomes the origin, and we know from Theorem 1, that there are no crossing periodic orbits for $\gamma_{R}+\gamma_{L} \leqslant 0$, while there is only one unstable crossing periodic orbit for $\gamma_{R}+\gamma_{L}>0$.

Assume that $b>0$ and $\gamma_{R} \leqslant 0$. From Theorem 2(c) we conclude that there is a unique stable crossing periodic orbit for $b \geqslant b_{C C}$ and that there are exactly two crossing periodic orbits with opposite stabilities for $b_{S N}<b<b_{C C}$. At the curve $b=b_{S N}\left(\gamma_{R}\right)$, a semi-stable crossing periodic orbits appears, so that there are no crossing limit cycles for $0<b<b_{S N}$, which is coherent with the non-existence of crossing periodic orbits when $b=0$. Note that for $b=b_{C C}$, the stable crossing limit cycle coexists with the unstable crossing critical cycle predicted by Proposition 2. 


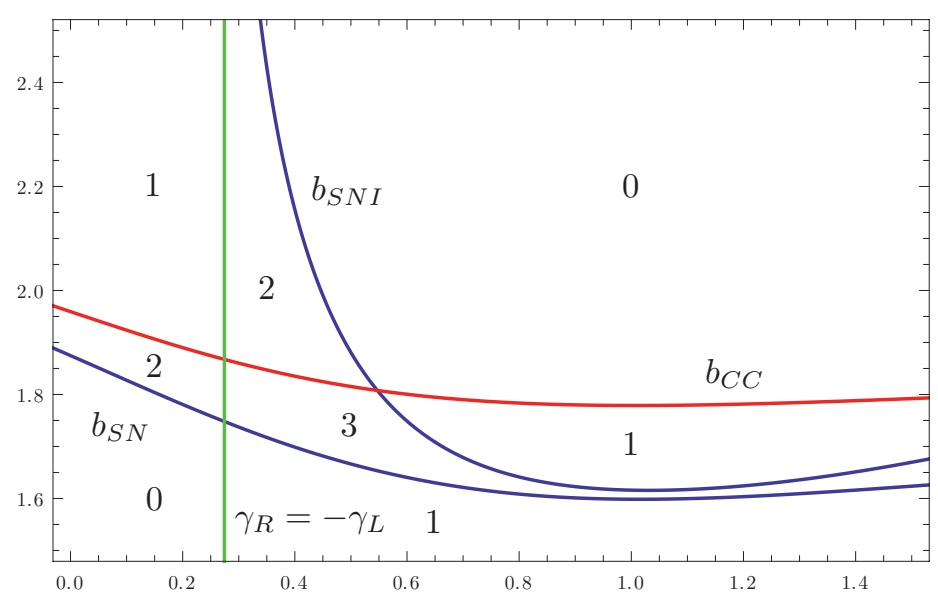

FiguRE 4. The zoomed rectangle of Figure 3 showing the curves $b_{C C}\left(\gamma_{R}\right), b_{S N}\left(\gamma_{R}\right)$ and $\gamma_{R}=-\gamma_{L}$ for the values $x_{L}=-1, x_{R}=-7$ and $\gamma_{L} \approx-0.27441$, in the plane $\left(\gamma_{R}, b\right)$. The digits in each region indicate the number of crossing limit cycles of the system.

Assume next that $b>0$ and $0<\gamma_{R}<-\gamma_{L}$. Now, we conclude from Theorem 4(a) the existence of at least one stable crossing periodic orbit for $b \geqslant b_{C C}$; from Theorem $5(\mathrm{a})$, we deduce the existence of at least two crossing limit cycles with opposite stabilities for $0<b_{C C}-b \ll 1$, and from Theorem 4(b) the non-existence of crossing periodic orbits for $0<$ $b<2 x_{L} \gamma_{L}$. Then, if we consider the simplest bifurcation set compatible with the quoted results, it could be claimed the existence of only one stable crossing periodic orbit for $b \geqslant b_{C C}$, exactly two crossing periodic orbits with opposite stabilities for $b_{S N}<b<b_{C C}$, and a saddle-node bifurcation of crossing periodic orbits at the curve $b=b_{S N}\left(\gamma_{R}\right)$. Finally, for $0<b<b_{S N}$, it can be also claimed the non-existence of crossing limit cycles, which would be again coherent with the non-existence of crossing periodic orbits when $b=0$. See Figure 4 , where the number of crossing limit cycles is shown in each open parameter region.

Assume now $\gamma_{L}+\gamma_{R}>0$, keeping $b>0$. For the case shown in Figures 3 and 4 , we see that both curves $b_{S N}$ and $b_{C C}$ persist for $\gamma_{R}>$ $-\gamma_{L}$ and that another curve $b_{S N I}$, existing only for $\gamma_{R}>-\gamma_{L}$, emerges tangentially from the line $\gamma_{R}=-\gamma_{L}$ at the point $\left(-\gamma_{L}, b_{\infty}\right)$. From Theorem 4(c) we deduce the existence of at least one unstable crossing 
periodic orbit for $0<b<b_{C C}$ and from Theorem 4 (d) we can assure the non-existence of crossing periodic orbits for $b$ big enough. From Theorem 5(b), we can assure the existence of at least three crossing limit cycles for $0<\gamma_{R}+\gamma_{L} \ll 1$ and $0<b_{C C}-b \ll 1$. Then, if we consider the simplest bifurcation set compatible with the quoted results, it could be claimed for the case of Figure 3 the non-existence of crossing limit cycles for $b>\max \left\{b_{S N I}, b_{C C}\right\}$, the existence of only one unstable crossing limit cycle in the regions $0<b<b_{S N}$ and in the one limited by the curves $b_{C C}$ and $b_{S N I}$, the existence of two crossing limit cycles in the region limited by the lines $\gamma_{L}+\gamma_{R}=0, b_{S N I}$ and $b_{C C}$, and finally the existence of three crossing limit cycles in the region $\gamma_{R}+\gamma_{L}>0$ and $b_{S N}<b<\min \left\{b_{C C}, b_{S N I}\right\}$. See Figures 3 and 4 , and note that the region with three limit cycles seems not bounded from the right.

Regarding the straight line $\gamma_{R}=-\gamma_{L}$ in Figures 3 and 4, from Theorem 4 (e) we see that for $b<b_{\infty}$ there appears a subcritical Hopf bifurcation at infinity, leading to the birth of a unstable crossing periodic orbit of big amplitude for $\gamma_{R}>-\gamma_{L}$. Analogously, for $b>b_{\infty}$ there appears a supercritical Hopf bifurcation at infinity, leading to the birth of a stable crossing periodic orbit of big amplitude for $\gamma_{R}<-\gamma_{L}$. Also, at the point $\left(-\gamma_{L}, b_{\infty}\right)$ we have a degenerate bifurcation point where another curve of bifurcation points corresponding to the collision of two limit cycles begins. The complete analysis of this Hopf bifurcation at infinity and its possible degeneracies will appear elsewhere.

We finish this section by noticing that the dynamical behavior for the case $b<0$ can be established by using an appropriate symmetry of Table 1. Effectively, by applying the transformation $\Pi_{1}$ to our system, we get a new system with $\gamma_{L}>0$ and $b>0$. Then from Theorems 2 and 3 we easily deduce the existence of a unique stable crossing periodic orbit only for $\gamma_{R}+\gamma_{L}<0$. Transferring this last result to our system we conclude the existence of a unique unstable crossing periodic orbit for $b<0$ and $\gamma_{L}<0$ only when $\gamma_{R}+\gamma_{L}>0$.

The rest of the paper is organized as follows. The Poincaré maps which constitute the main tools used through the analysis are studied in Section 3. Finally, in Section 4 the proofs of the main results are given.

\section{Analysis of the Poincaré return map}

We start by giving, the expression of the solutions in each linearity zone. For $x \neq 0$, system (5) can be written as

$$
\dot{\mathbf{x}}=\left(\begin{array}{cc}
2 \gamma & -1 \\
1+\gamma^{2} & 0
\end{array}\right) \mathbf{x}-\left(\begin{array}{c}
-\sigma \\
\left(1+\gamma^{2}\right) \bar{x}
\end{array}\right)
$$


for appropriate values of parameters $\gamma, \sigma$ and $\bar{x}$. Its solutions have the form,

$$
\left(\begin{array}{l}
x(t)-\bar{x} \\
y(t)-\bar{y}
\end{array}\right)=e^{\gamma t}\left(\begin{array}{cc}
\cos t+\gamma \sin t & -\sin t \\
\left(1+\gamma^{2}\right) \sin t & \cos t-\gamma \sin t
\end{array}\right)\left(\begin{array}{c}
x(0)-\bar{x} \\
y(0)-\bar{y}
\end{array}\right),
$$

where $\bar{y}=2 \gamma \bar{x}+\sigma$.

We also introduce the auxiliary function, see [1]

$$
\varphi_{\gamma}(t)=1-e^{\gamma t}(\cos t-\gamma \sin t) \text {. }
$$

Function $\varphi_{\gamma}$ has the following symmetry properties

$$
\varphi_{-\gamma}(t)=\varphi_{\gamma}(-t), \quad \varphi_{-\gamma}(t)=\varphi_{\gamma}(-t), \quad \forall \gamma, t \in \mathbb{R},
$$

and the graph shown in Figure 5. Note that function $\varphi_{\gamma}$ has relative maxima at $t= \pm \pi$ and for $\gamma>0$ a first positive zero for a certain value $\hat{t} \in(\pi, 2 \pi)$.

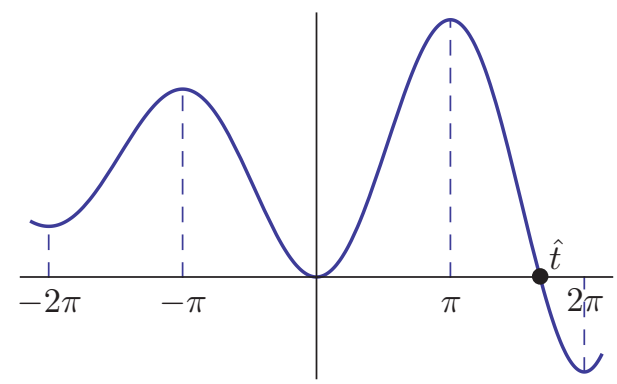

FIGURE 5. The graph of function $\varphi_{\gamma}(\cdot)$ for a positive value of $\gamma$.

Next, we introduce some Poincaré maps which are our main tool in looking for crossing periodic orbits. For system (5), under the focus hypotheses, the direction of the flow at the line $x=0$ assures that orbits starting at points $(0, y)$ with $y>0$ and big enough go into the zone $S^{-}$ until it again reaches $\Sigma$ at a point $\left(0, y_{1}\right)$ with $y_{1} \leqslant 0$ after a time $t_{L}$. Thus, we define a left Poincaré map $P_{L}$ as $y_{1}=P_{L}(y) \leqslant 0$.

In order to integrate system (5) in the left zone we must put $\sigma=0$ and $\bar{x}=x_{L}$ in (9). If $\gamma_{L}=0$, then from (9) we explicitly obtain the map $P_{L}$,

$$
P_{L}(y)=-y, \quad y \geqslant 0 .
$$


If $x_{L}<0$, then the point $(0,0)$ is a visible left tangency point and from (9) we obtain a parametric representation of the left Poincaré map $P_{L}$,

$$
y=\frac{e^{-\gamma_{L} t_{L}} \varphi_{\gamma_{L}}\left(t_{L}\right)}{\sin t_{L}} x_{L}, \quad P_{L}(y)=-\frac{e^{\gamma_{L} t_{L}} \varphi_{-\gamma_{L}}\left(t_{L}\right)}{\sin t_{L}} x_{L},
$$

where $t_{L} \in\left(\pi, \hat{t}_{L}\right)$, being $\varphi_{\left|\gamma_{L}\right|}\left(\hat{t}_{L}\right)=0$. In this case the orbits must go around the real equilibrium $\mathbf{e}_{L}$ located in $S^{-}$.

Direct computations from (11) show that the two first derivatives of the left Poincaré map when $\gamma_{L} \neq 0$ are given by

$$
\begin{aligned}
& P_{L}^{\prime}(y)=-\frac{\varphi_{\gamma_{L}}\left(t_{L}\right)}{\varphi_{-\gamma_{L}}\left(t_{L}\right)}=\frac{y}{P_{L}(y)} e^{2 \gamma_{L} t_{L}}<0, \\
& P_{L}^{\prime \prime}(y)=2 x_{L}^{2}\left(1+\gamma_{L}^{2}\right) \frac{\sinh \gamma_{L} t_{L}-\gamma_{L} \sin t_{L}}{P_{L}^{3}(y)} e^{3 \gamma_{L} t_{L}} .
\end{aligned}
$$

Integrating the system in the zone $S^{+}$from the point $(0, z)$ with $z<b$, after a time $t_{R}$ we arrive at the point $\left(0, z_{1}\right)$ with $z_{1}>b$, and so we can define the right Poincaré map $P_{R}$ as $z_{1}=P_{R}(z)>b$.

In the trivial case $\gamma_{R}=0$, from (9) we explicitly obtain

$$
P_{R}(z)=-z+2 b, \quad z \leqslant b .
$$

If $x_{R}<0$, the point $(0, b)$ is a right invisible tangency point and the parametric representation of the right Poincaré map is given by,

$$
z=b+\frac{e^{-\gamma_{R} t_{R}} \varphi_{\gamma_{R}}\left(t_{R}\right)}{\sin t_{R}} x_{R}, \quad P_{R}(z)=b-\frac{e^{\gamma_{R} t_{R}} \varphi_{-\gamma_{R}}\left(t_{R}\right)}{\sin t_{R}} x_{R}
$$

where $t_{R} \in(0, \pi)$.

Analogously, the two first derivatives of the right Poincaré map when $\gamma_{R} \neq 0$ are given by

$$
\begin{aligned}
& P_{R}^{\prime}(z)=\frac{z-b}{P_{R}(z)-b} e^{2 \gamma_{R} t_{R}}<0, \\
& P_{R}^{\prime \prime}(z)=2 x_{R}^{2}\left(1+\gamma_{R}^{2}\right) \frac{\sinh \gamma_{R} t_{R}-\gamma_{R} \sin t_{R}}{\left(P_{R}(z)-b\right)^{3}} e^{3 \gamma_{R} t_{R}} .
\end{aligned}
$$

Finally, as the main tool in looking for crossing periodic orbits, we define the Poincaré map $P$ as the composition $P=P_{R} \circ P_{L}$, to be defined for points $(0, y)$ with $y>0$ and big enough.

Next, we establish some properties of Poincaré maps when system (5) has only one equilibria which is located in the zone $x<0$, that is when $x_{L}<0$ and $x_{R}<0$. We begin by considering the left Poincaré map $P_{L}$. 
Proposition 3 (The map $P_{L}$ ). Assume that $x_{L}<0$ in system (5). Then the following statements are true for the left Poincaré map $P_{L}$.

(a) If $\gamma_{L}=0$, then $P_{L}(y)=-y$ for all $y \geqslant 0$. If $\gamma_{L} \neq 0$, then $P_{L}$ is defined in (11) for $\pi<t \leqslant \hat{t}_{L}$ being $\varphi_{\left|\gamma_{L}\right|}\left(\hat{t}_{L}\right)=0$.

(b) If $\gamma_{L}<0$, then we define $\hat{y}=y_{0}\left(\hat{t}_{L}\right)>0$, and we have $P_{L}(\hat{y})=0$,

$$
P_{L}:[\hat{y}, \infty) \rightarrow(-\infty, 0] \text { and } \lim _{y \rightarrow \hat{y}^{+}} P_{L}^{\prime}(y)=-\infty .
$$

(c) If $\gamma_{L}>0$, then we define $\hat{y}_{1}=y_{1}\left(\hat{t}_{L}\right)<0$, and we have $P_{L}(0)=\hat{y}_{1}$,

$$
P_{L}:[0, \infty) \rightarrow\left(-\infty, \hat{y}_{1}\right] \quad \text { and } \lim _{y \rightarrow 0^{+}} P_{L}^{\prime}(y)=0
$$

(d) If $\gamma_{L} \neq 0$, then we have $P_{L}^{\prime}(y)<0$ for all $y$ where map $P_{L}$ is defined,

$$
\lim _{y \rightarrow \infty} P_{L}^{\prime}(y)=-e^{\gamma_{L} \pi}, \quad \operatorname{sign} P_{L}^{\prime \prime}(y)=-\operatorname{sign} \gamma_{L},
$$

and map $P_{L}$ has the asymptote $A_{L}$ given by

$$
A_{L}(y)=-e^{\gamma_{L} \pi} y+2 x_{L} \gamma_{L}\left(1+e^{\gamma_{L} \pi}\right)
$$

with $\operatorname{sign}\left(A_{L}(y)-P_{L}(y)\right)=\operatorname{sign}\left(\gamma_{L}\right)$.

Proof: Statement (a) follows from (10) and (11). To prove statement (b) assume $\gamma_{L}<0$, then $\pi<t_{L}<\hat{t}_{L}$ and from (12) we get

$$
\lim _{y \rightarrow \hat{y}^{+}} P_{L}^{\prime}(y)=\lim _{t_{L} \rightarrow \hat{t}_{L}^{+}} P_{L}^{\prime}(y)=-\infty .
$$

The proof of statement (c) is analogous to the proof of statement (b).

If $\gamma_{L} \neq 0$, then

$$
\lim _{y \rightarrow \infty} P_{L}^{\prime}(y)=\lim _{t_{L} \rightarrow \pi^{-}} P_{L}^{\prime}(y)=-e^{\gamma_{L} \pi} .
$$

Since $\operatorname{sign}\left(\sinh \gamma_{L} t_{L}-\gamma_{L} \sin t_{L}\right)=\operatorname{sign} \gamma_{L}$, from (13) it is easy to see that $\operatorname{sign} P_{L}^{\prime \prime}(y)=-\operatorname{sign} \gamma_{L}$. The computation of the asymptote is straightforward and the proof is finished.

Here, we see that the proof of Proposition 1 directly follows from the properties of left Poincaré map given in Proposition 3 and the discussion done above the statement of Proposition 1.

The following proposition about the right Poincaré map is similar and it is given without proof.

Proposition 4 (The map $P_{R}$ ). Assuming $x_{R}<0$ in system (5), the following statements hold for the right Poincaré map $P_{R}$. 
(a) If $\gamma_{R}=0$ then, $P_{R}(y)=-y+2 b$ for all $y \leqslant b$.

(b) If $\gamma_{R} \neq 0$ then the right Poincaré map $P_{R}$ given in (15) is defined for all $y \leqslant b$, where $t \in(0, \pi)$; in particular we have $P_{R}(b)=b$. Its two first derivatives satisfy

$$
P_{R}^{\prime}(b)=-1, \quad P_{R}^{\prime}(y)<0, \quad \lim _{y \rightarrow-\infty} P_{R}^{\prime}(y)=-e^{\gamma_{R} \pi}
$$

and its second derivative satisfies $\operatorname{sign} P_{R}^{\prime \prime}(y)=\operatorname{sign} \gamma_{R}$.

(c) The map $P_{R}^{-1}$ has the asymptote $A_{R^{-1}}$ given by

$$
A_{R^{-1}}(y)=-e^{-\gamma_{R} \pi} y+\left(b+2 x_{R} \gamma_{R}\right)\left(1+e^{-\gamma_{R} \pi}\right)
$$

and satisfies $\operatorname{sign}\left(P_{R}^{-1}(y)-A_{R^{-1}}(y)\right)=\operatorname{sign}\left(\gamma_{R}\right)$.

As a consequence of the above results we obtain some properties for the complete Poincaré map. In Figure 6 the left and right Poincaré maps are shown for different values of parameters $\gamma_{L}$ and $\gamma_{R}$.
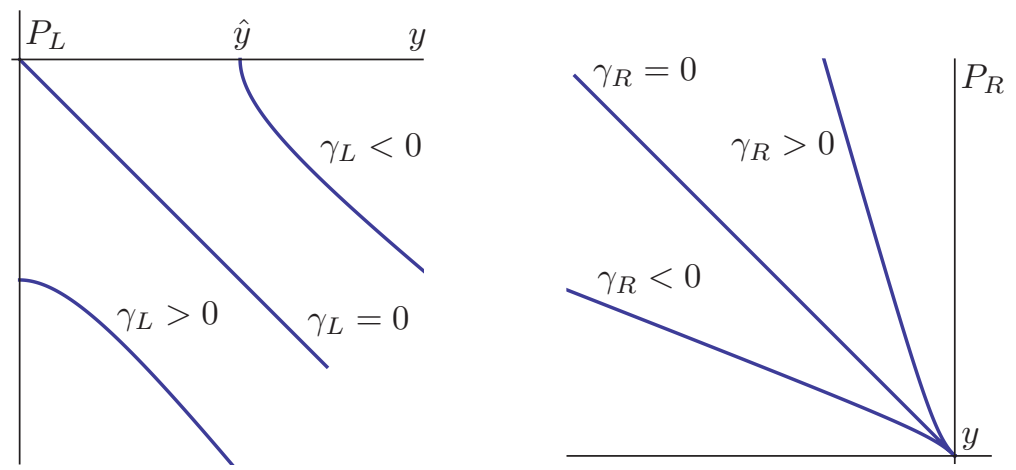

Figure 6 . The left Poincaré map for different values of parameter $\gamma_{L}$ and the right Poincaré map for different values of parameter $\gamma_{R}$ and $b=0$.

Proposition 5 (The full Poincaré map). Assuming $x_{L}<0$ and $x_{R}<0$ in system (5), the following statements hold.

(a) The Poincaré map $P$ is well defined for $y \geqslant y_{P} \geqslant 0$ where, as defined in Proposition 3(b), $y_{P}=\hat{y}$ for $\gamma_{L}<0$ and $y_{P}=0$ when $\gamma_{L} \geqslant 0$.

(b) If $\gamma_{R}=0$, then $P(y)=-P_{L}(y)+2 b$. If in addition $\gamma_{L}=0$, then $P(y)=y+2 b$.

(c) If $\gamma_{L}=0$, then $P(y)=P_{R}(-y)$ and $\operatorname{sign} P^{\prime \prime}(y)=-\operatorname{sign} \gamma_{R}$. 
(d) For all $y>y_{P}$, the derivative of map $P$ is given by

$$
P^{\prime}(y)=\frac{y}{P_{L}(y)} \frac{P_{L}(y)-b}{P(y)-b} e^{2\left(\gamma_{R} t_{R}+\gamma_{L} t_{L}\right)}
$$

and satisfies

$$
\lim _{y \rightarrow \infty} P^{\prime}(y)=e^{\left(\gamma_{R}+\gamma_{L}\right) \pi}, \quad \lim _{y \rightarrow y_{P}^{+}} P^{\prime}(y)= \begin{cases}\infty, & \text { if } \gamma_{L}<0 \\ 1, & \text { if } \gamma_{L}=0 \\ 0, & \text { if } \gamma_{L}>0\end{cases}
$$

Proof: Statements (a), (b), and (c) directly follows from Propositions 3 and 4. Statement (d) can be easily deduced from Propositions 3, 4 and the chain rule.

Remark 2. It is worth noting that from Proposition 5(d), it is direct to deduce the stability of the point at infinity. More precisely, if $\gamma_{R}+\gamma_{L}<0$, then the point at infinity is unstable while is stable for $\gamma_{R}+\gamma_{L}>0$.

\section{Proof of main results}

First, we review a necessary condition for the existence of crossing periodic orbits. A crossing periodic orbit $\Gamma$ has exactly two points at the $y$-axis, namely the two points $\left(0, y_{L}\right)$ and $\left(0, y_{U}\right)$ with $y_{L}<0 \leqslant b<$ $y_{U}=y_{L}+h$, with $h>0$. We can define, by removing the two crossing points, the left open $\operatorname{arc} \Gamma_{L}=\Gamma \cap S^{-}$, the right open $\operatorname{arc} \Gamma_{R}=\Gamma \cap S^{+}$, and the oriented segments,

$$
I_{L}=\left\{(x, y): x=0, \quad y=(1-\mu) y_{L}+\mu y_{U}, \quad 0 \leqslant \mu \leqslant 1\right\},
$$

and

$$
I_{R}=\left\{(x, y): x=0, \quad y=\mu y_{L}+(1-\mu) y_{U}, \quad 0 \leqslant \mu \leqslant 1\right\} .
$$

Since $\Gamma_{L} \cup I_{L}$ is a closed Jordan curve, its interior $\Omega_{L}=\operatorname{int}\left\{\Gamma_{L} \cup I_{L}\right\}$ and the value $\sigma_{L}=$ area $\left(\Omega_{L}\right)$ are well defined. Analogously, it can be considered $\Omega_{R}=\operatorname{int}\left\{\Gamma_{R} \cup I_{R}\right\}$ and $\sigma_{R}=$ area $\left(\Omega_{R}\right)$. Then, according to Proposition 3.6 of $[\mathbf{9}]$, if system (5) has a crossing periodic orbit passing through the points $\left(0, y_{L}\right)$ and $\left(0, y_{L}+h\right)$ where $h>0$, then

$$
2 \gamma_{L} \sigma_{L}+2 \gamma_{R} \sigma_{R}+b h=0 .
$$

Remark 3. We remark that in the two cases (i) $\gamma_{L}>0, \gamma_{R}>0, b>0$; and (ii) $\gamma_{L} \gamma_{R} \geqslant 0, \gamma_{L}+\gamma_{R} \neq 0, b=0$, the equality (18) cannot be fulfilled and so, system (5) cannot have crossing limit cycles.

In the following we will tackle the proofs of the different assertions given in Section 2. Next we consider the proof of Theorem 1. 
Proof of Theorem 1: (a) See Remark 3.

(b) From Proposition 5(b) we have $P(y)=y$ for $y \geqslant 0$, and the statement follows.

When the divergence are opposite, that is $\gamma_{R} \gamma_{L}<0$, then by using the transformation $\Pi_{1}$ if necessary, see Table 1 , we see that it is enough to consider $\gamma_{L}<0$ and $\gamma_{R}>0$.

Firstly, we will prove that Poincaré map $P$ has at most a fixed point. Assuming $\gamma_{L}<0$, from Proposition 5(a), a value $\hat{y}>0$ exists such that the Poincaré map is defined for $y \geqslant y_{P}=\hat{y}$ and $P(\hat{y})=0$. For $y \geqslant y_{P}$, we introduce the function $\nu(y)=\gamma_{L} t_{L}+\gamma_{R} t_{R}$, with $\pi<t_{L}<\hat{t}_{L}$ and $0<t_{R}<\pi$, where $t_{L}$ is the phase needed in (11) to obtain $P_{L}$ and $t_{R}$ is the corresponding phase in (15) to determine $z=P_{L}(y)$ and $P_{R}(z)=P(y)$. In this case, when $y$ increases $t_{L}$ becomes smaller and $t_{R}$ becomes bigger and so the function $\nu(y)$ is increasing.

From Proposition 5(d), taking into account that $b=0$ the derivative of the Poincaré map at a fixed point $\bar{y}$, if any, reduces to $P^{\prime}(\bar{y})=e^{2 \nu(\bar{y})}$. Suppose now that map $P$ has several fixed points and let $\bar{y}_{1}$ be the smallest one. Then it is easy to see that $P^{\prime}\left(\bar{y}_{1}\right) \geqslant 1$, and so $\nu\left(\bar{y}_{1}\right) \geqslant 0$. Taking the next fixed point $\bar{y}_{2}>\bar{y}_{1}$, the only possibility is

$$
\nu\left(\bar{y}_{1}\right)=0, \quad 1=P^{\prime}\left(\bar{y}_{1}\right)<P^{\prime}\left(\bar{y}_{2}\right)=e^{2 \nu\left(\bar{y}_{2}\right)}
$$

because two consecutive fixed points with derivatives greater than 1 are not allowed. In this situation, for points $y \in\left(\bar{y}_{1}, \bar{y}_{2}\right)$ we have $y>P(y)$ and $\nu(y)>0$, so putting $b=0$ in Proposition $5(\mathrm{~d})$ we get $P^{\prime}(y)>1$ for all $y \in\left(\bar{y}_{1}, \bar{y}_{2}\right)$, which leads to a contradiction with the mean value theorem in such interval. Therefore, the map $P$ has at most only one fixed point.

(c) As mentioned before, we can assume without loss of generality $\gamma_{L}<0$ and so $\gamma_{L}+\gamma_{R} \leqslant 0$. Suppose also that the Poincaré map has a fixed point $\bar{y}$, then we must have $\nu(\bar{y}) \geqslant 0$. But in this case, we have

$$
\nu(\bar{y})=\gamma_{L} t_{L}+\gamma_{R} t_{R} \leqslant \gamma_{L}\left(t_{L}-t_{R}\right)<0,
$$

because $0<\gamma_{R} \leqslant-\gamma_{L}$ and $t_{R}<\pi<t_{L}$. We get a contradiction, so the map $P$ has no fixed points and there are no crossing periodic orbits.

(d) It suffices to assume $\gamma_{L}+\gamma_{R}>0$. Since $P(\hat{y})=0$ and from Proposition $5(\mathrm{~d})$ we have $\lim _{y \rightarrow \infty} P^{\prime}(y)>1$, by using the mean value theorem adequately, we deduce that $P(y)>y$ when $y$ is big enough. Consequently, from the intermediate value theorem there exists a value $\bar{y}$ with 
$P(\bar{y})=\bar{y}$ and $P^{\prime}(\bar{y})>1$, i.e. system (5) has only one crossing limit cycle, which is unstable.

In the following, the existence of periodic orbits in system (5) for $b>0$ is considered. Note that the left Poincaré map does not depends upon the parameter $b$, but both Poincaré maps $P_{R}$ and $P_{R}^{-1}$ depend on the parameter $b$ in a rather specific way. In fact, if we make explicit the dependence on $b$ in the notation, from (14) and (15) we see that

$$
P_{R}^{-1}(y ; b)=b+P_{R}^{-1}(y-b ; 0) .
$$

In short, the graph of $P_{R}^{-1}(y ; b)$ is just a translation of the graph of $P_{R}^{-1}(y ; 0)$ by the vector $(b, b)$, see Figure 7 .

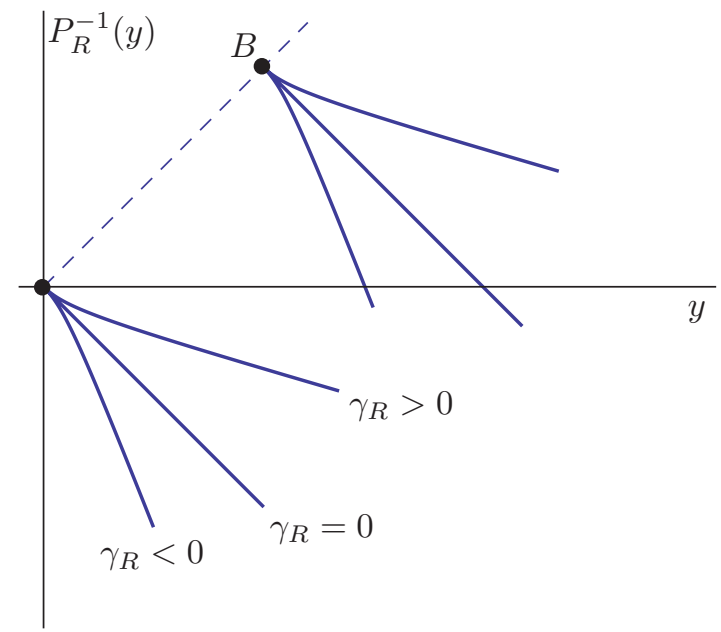

Figure 7. The inverse half Poincaré map $P_{R}^{-1}$ for $b=$ 0 and several values of parameter $\gamma_{R}$. To obtain the map $P_{R}^{-1}$ for $b>0$, it suffices to translate the graphs to the point $B$, which has the coordinates $(b, b)$.

If $\bar{y}$ is a fixed point of the Poincaré map $P$, then $P(\bar{y})=P_{R}\left(P_{L}(\bar{y}) ; b\right)=$ $\bar{y}$, and so $P_{L}(\bar{y})=P_{R}^{-1}(\bar{y} ; b)$. Hence the existence of crossing periodic orbits is equivalent to the existence of zeroes with $y>y_{P}$ of the function

$$
\Psi(y ; b)=P_{R}^{-1}(y ; b)-P_{L}(y),
$$

well defined when both $y \geqslant y_{P}$ and $y \geqslant b$, where $y_{P} \geqslant 0$ satisfies $y_{P}=\hat{y}$ for $\gamma_{L}<0$ and $y_{P}=0$ otherwise. 
If we use ' to denote the derivatives with respect to the variable $y$, the two first derivatives of the function $\Psi$ when $y \neq \hat{y}$ read as

$$
\begin{aligned}
\Psi^{\prime}(y ; b) & =\frac{1}{\left(P_{R}^{\prime} \circ P_{R}^{-1}\right)(y ; b)}-P_{L}^{\prime}(y), \\
\Psi^{\prime \prime}(y ; b) & =-\frac{\left(P_{R}^{\prime \prime} \circ P_{R}^{-1}\right)(y ; b)}{\left(P_{R}^{\prime} \circ P_{R}^{-1}\right)^{3}(y ; b)}-P_{L}^{\prime \prime}(y) .
\end{aligned}
$$

In the next lemma some properties of the function $\Psi$ are introduced.

Lemma 1. Given $\gamma_{R}, \gamma_{L}$ and assuming $a_{L}<0, a_{R}<0, b>0$ for the function $\Psi$ defined in (20) the following statements hold.

(a) If $\Psi(y ; b) \neq 0$, then $\Psi(y ; b)(P(y)-y)>0$.

(b) If $\Psi(\bar{y} ; b)=0$, then $P(\bar{y})=\bar{y}$. If in addition $\bar{y} \neq \hat{y}$ and $\Psi$ is decreasing (respectively increasing) with respect to variable $y$ at the point $\bar{y}$, then $\bar{y}$ corresponds to a stable (respectively unstable) crossing periodic orbit. In particular, if $\Psi^{\prime}(\bar{y} ; b)<0$, then $\bar{y}$ corresponds to a stable crossing periodic orbit (respectively, unstable for $\left.\Psi^{\prime}(\bar{y} ; b)>0\right)$. Furthermore, we have

$$
\delta_{\infty}=\lim _{y \rightarrow \infty} \Psi^{\prime}(y ; b)=\left(e^{\left(\gamma_{R}+\gamma_{L}\right) \pi}-1\right) e^{-\gamma_{R} \pi} .
$$

(c) The function $\Psi$ and its derivative with respect to the parameter $b$ satisfy the inequalities

$$
\Psi(y ; b)>b+\Psi(y ; 0), \quad \frac{\partial \Psi(y ; b)}{\partial b}=\frac{\partial P_{R}^{-1}(y ; b)}{\partial b}>1 .
$$

(d) If $\gamma_{L} \gamma_{R} \geqslant 0$ and $y \neq \hat{y}$, then $\operatorname{sign} \Psi^{\prime \prime}(y ; b)=\operatorname{sign}\left(\gamma_{L}+\gamma_{R}\right)$ and so function $\Psi$ has at most two zeroes.

Proof: (a) If $\Psi(y ; b)>0$, then $P_{L}(y)<P_{R}^{-1}(y ; b)$ and applying to this inequality $P_{R}$, which is decreasing, we get $P(y)>y$. Analogously, when $\Psi(y ; b)<0$, we get $P(y)<y$.

(b) If $\Psi(\bar{y} ; b)=0$, then it directly follows $P(\bar{y})=\bar{y}$. If the function $\Psi$ is decreasing (respectively, increasing) then $h(p)=P(p)-p$ is also decreasing (respectively, increasing) and $\bar{p}$ corresponds to a stable (respectively unstable) crossing periodic orbit. The other assertions of this statement are direct.

(c) From (19) we have $\Psi(y ; b)=b+P_{R}^{-1}(y-b, 0)-P_{L}(y)$. Since the map $P_{R}^{-1}$ is decreasing and $b>0$, we have

$$
\Psi(y ; b)>b+P_{R}^{-1}(y, 0)-P_{L}(y)=b+\Psi(y ; 0),
$$


and the derivative with respect to the parameter $b$ satisfies,

$$
\frac{\partial \Psi(y ; b)}{\partial b}=1-\left(P_{R}^{-1}\right)^{\prime}(y-b ; 0)>1
$$

(d) From Proposition $3(\mathrm{~d})$ we have $\operatorname{sign} P_{L}^{\prime \prime}(y)=-\operatorname{sign} \gamma_{L}$ for $y \neq \hat{y}$. From Proposition 4(b) we also have $\operatorname{sign} P_{R}^{\prime \prime}(y)=\operatorname{sign} \gamma_{R}$, and having in mind that $P_{R}^{\prime}(y)<0$, the conclusion follows from $(21)$.

Of course, the function $\Psi$ depends on the values $\gamma_{R}$ and $\gamma_{L}$. When necessary, we will introduce the explicit dependence on the parameter $\gamma_{R}$, keeping fixed the value of $\gamma_{L}$ if the contrary it is not stated, so that we can use the notation $\Psi\left(y ; b ; \gamma_{R}\right)$.

As the function $\Psi$ is real analytic, we can assure that, in every compact interval where the function $\Psi$ has opposite signs at its endpoints, its number of zeroes is finite and so, there exists at least one zero where the function $\Psi$ is strictly monotone, either increasing or decreasing. More precisely, if $\Psi\left(y_{1} ; \bar{b}, \bar{\gamma}_{R}\right) \Psi\left(y_{2} ; \bar{b}, \bar{\gamma}_{R}\right)<0$, then there exists at least a value $\bar{y}$ with $y_{1}<\bar{y}<y_{2}$, such that $\Psi\left(\bar{y} ; \bar{b}, \bar{\gamma}_{R}\right)=0$, and $\Psi$ is a monotone function in the variable $y$ at the point $\bar{y}$. Therefore, if $\Psi\left(y_{1} ; \bar{b}, \bar{\gamma}_{R}\right)>\Psi\left(y_{2} ; \bar{b}, \bar{\gamma}_{R}\right)$ we can assure the existence of a stable crossing limit cycle, and when $\Psi\left(y_{1} ; \bar{b}, \bar{\gamma}_{R}\right)<\Psi\left(y_{2} ; \bar{b}, \bar{\gamma}_{R}\right)$ the existence of an unstable crossing limit cycle.

Here, we state an important remark about persistence of crossing periodic orbits.

Remark 4. Assume for system (5) the existence of a crossing periodic orbit corresponding to the condition $\Psi\left(\bar{y}, \bar{b}, \bar{\gamma}_{R}\right)=0$ with $\bar{y} \neq \hat{y}$ and that for certain values $\alpha>0$ and $\beta>0$ the inequality $\Psi\left(\bar{y}-\alpha ; \bar{b}, \bar{\gamma}_{R}\right) \Psi(\bar{y}+$ $\left.\beta ; \bar{b}, \bar{\gamma}_{R}\right)<0$ holds. By the continuity of the function $\Psi$ with respect to the parameters $b$ and $\gamma_{R}$, we must have for $\left|\varepsilon_{1}\right|$ and $\left|\varepsilon_{2}\right|$ small enough

$$
\operatorname{sign} \Psi\left(\bar{y}-\alpha ; \bar{b}+\varepsilon_{1}, \bar{\gamma}_{R}+\varepsilon_{2}\right)=\operatorname{sign} \Psi\left(\bar{y}-\alpha ; \bar{b}, \bar{\gamma}_{R}\right)
$$

and

$$
\operatorname{sign} \Psi\left(\bar{y}+\beta ; \bar{b}+\varepsilon_{1}, \bar{\gamma}_{R}+\varepsilon_{2}\right)=\operatorname{sign} \Psi\left(\bar{y}+\beta ; \bar{b}, \bar{\gamma}_{R}\right) .
$$

Then, there exists at least a value $\varepsilon_{3}$ such that $\Psi\left(\bar{y}+\varepsilon_{3} ; \bar{b}+\varepsilon_{1}, \bar{\gamma}_{R}+\varepsilon_{2}\right)=$ 0 , and with the function $\Psi\left(y ; \bar{b}+\varepsilon_{1}, \bar{\gamma}_{R}+\varepsilon_{2}\right)$ having the same type of monotonicity at the points $\bar{y}$ and $\bar{y}+\varepsilon_{3}$. Hence, the assumed crossing periodic orbit persists with the same stability under a sufficiently small perturbation in parameters $b$ and $\gamma_{R}$.

Next, we will analyze the persistence of the crossing critical cycle, that is the persistence of the zeroes $\Psi(\hat{y} ; b)=0$. First we present a technical lemma. 
Lemma 2. If $x_{R}<0$ then for every value $y_{R}>0$ there exists a unique value $0<b_{R}<y_{R}$ such that $P_{R}^{-1}\left(y_{R} ; b_{R}\right)=0$.

Proof: Let us introduce the function $\varphi(b)=P_{R}^{-1}\left(y_{R} ; \gamma_{R}, b\right)$, defined for every value of $\gamma_{R}$ and $0 \leqslant b \leqslant y_{R}$. By considering the endpoints of the domain of the function $\varphi$ we have $\varphi(0)<0$ and $\varphi\left(y_{R}\right)=y_{R}>$ 0 , so there exists a value $b_{R}$ with $0<b_{R}<y_{R}$ such that $\varphi\left(b_{R}\right)=$ $P_{R}^{-1}\left(y_{R} ; \gamma_{R}, b_{R}\right)=0$. Since funtion $\varphi$ is increasing, see Lemma $1(\mathrm{c})$, we conclude the uniqueness of the value $b_{R}$.

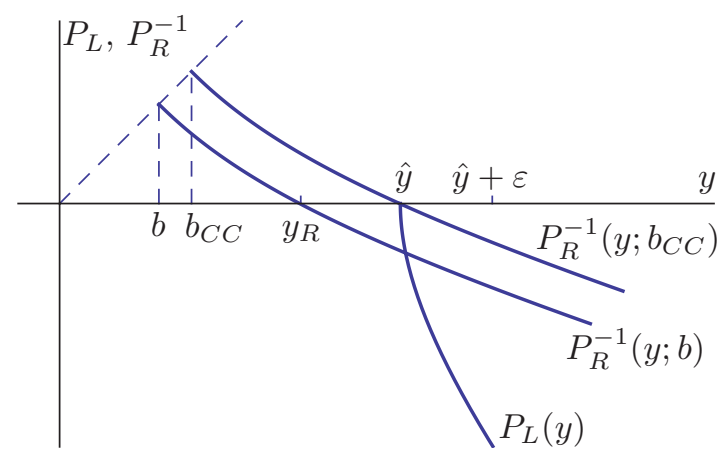

Figure 8 . The map $P_{L}$ for $\gamma_{L}<0$ and the map $P_{R}^{-1}$ in a neighborhood of $b=b_{C C}$. For $b=b_{C C}$ we have $P_{L}(\hat{y})=P_{R}^{-1}(\hat{y})=0$ and there exists a crossing critical cycle. For $b_{C C}-\varepsilon<b<b_{C C}$ we have $\Psi(\hat{y} ; b)<0$, while $\Psi(\hat{y}+\varepsilon ; b)>0$ and one unstable crossing limit cycle exists.

Proof of Proposition 2: From Proposition 3(b) there exists a value $\hat{y}>$ 0 , which does not depend on parameter $b$, with $P_{L}(\hat{y})=0$. By Lemma 2 , we can select a unique value $b_{C C}=b_{C C}\left(\gamma_{R}\right)$ with $0<b_{C C}\left(\gamma_{R}\right)<\hat{y}$ such that $P_{R}^{-1}\left(\hat{y}, \gamma_{R}, b_{C C}\left(\gamma_{R}\right)\right)=0$. Taking $\gamma_{R}=0$ in (15) it is direct to obtain $b_{C C}(0)=\hat{y} / 2$.

Clearly, we have $\Psi\left(\hat{y} ; b_{C C}\right)=P_{R}^{-1}\left(\hat{y}, b_{C C}\right)=P_{L}(\hat{y})=0$. This configuration corresponds to a critical crossing cycle. Furthermore, it is easy to see that the orbits living in the interior of the crossing critical cycle evolve toward the origin while from Proposition 5 we get $\lim _{y \rightarrow \hat{y}^{+}} P^{\prime}(y)=-\infty$. Hence this critical crossing cycle must be unstable. 
Now, we will prove the existence of a unstable limit cycle for $0<b_{C C}-$ $b \ll 1$. From Proposition 4 (b), there exists a value $K=\min \left\{-1,-e^{-\gamma_{R} \pi}\right\}$ such that $K \leqslant\left(P_{R}^{-1}\right)^{\prime}(y ; b)<0$ for all $y>b$. Take $\varepsilon>0$ and $b$ with $0<b_{C C}-\varepsilon<b \leqslant b_{C C}$, then from Lemma 2, there exists a unique value $y_{R}$ with $b<y_{R} \leqslant \hat{y}$ and $P_{R}^{-1}\left(y_{R} ; b\right)=0$. From the intermediate value theorem we get for all $y>y_{R}$,

$$
P_{R}^{-1}(y ; b)=P_{R}^{-1}(y ; b)-P_{R}^{-1}\left(y_{R} ; b\right)=\left(P_{R}^{-1}(\xi ; b)\right)^{\prime}\left(y-y_{R}\right) \geqslant K\left(y-y_{R}\right) .
$$

From Proposition $3(\mathrm{~b})$ we have $\lim _{y \rightarrow \hat{y}^{+}} P_{L}^{\prime}(y)=-\infty$, and we can assure that for all $\varepsilon>0$ there exists $M>0$ with $M+K>0$, such that $\hat{y}<y \leqslant \hat{y}+\varepsilon$ implies

$$
\frac{P_{L}(y)-P_{L}(\hat{y})}{y-\hat{y}}=\frac{P_{L}(y)}{y-\hat{y}}<-M,
$$

since $P_{L}(\hat{y})=0$. So we get

$$
-P_{L}(y)>M(y-\hat{y}) \text { for all } \hat{y}<y \leqslant \hat{y}+\varepsilon .
$$

Then $\Psi(\hat{y} ; b)<0$, while from $(23)$ and $(24)$ we have

$$
\begin{aligned}
\Psi(\hat{y}+\varepsilon ; b)=P_{R}^{-1}(\hat{y}+\varepsilon ; b)-P_{L}(\hat{y}+\varepsilon) & >K\left(\hat{y}+\varepsilon-y_{R}\right) \\
& +M \varepsilon>(K+M) \varepsilon>0
\end{aligned}
$$

so there exists at least a value $\bar{y}$ with $\hat{y}<\bar{y}<\hat{y}+\varepsilon$ and $\Psi(\bar{y} ; b)=0$. Moreover, it is easy to see that $P^{\prime}(\bar{y})>1$, so $\bar{y}$ corresponds to a unstable crossing limit cycle.

Remark 5. We remark that under the conditions of Proposition 2, we have seen from $(25)$ that there exists $\varepsilon>0$ such that $\Psi\left(\hat{y}+\varepsilon ; b_{C C}\right)>0$.

Here, the proof of Theorem 2 follows.

Proof of Theorem 2: (a) See Remark 3.

(b) If $\gamma_{L}=0$, the only equilibrium point is a center. If additionally $\gamma_{R}<0$, from Proposition 5(c) we get $P^{\prime \prime}(y)>0$ always. Since $P(0)=$ $P_{R}(0)>b$ and $\lim P_{y \rightarrow \infty}^{\prime}(y)<1$, from the concavity of map $P$ we conclude the existence of a unique point $\bar{y}$ with $P(\bar{y})=\bar{y}$. Moreover, as $P^{\prime}(\bar{y})<1$, the corresponding crossing periodic orbit is stable.

(c) Since $\gamma_{R} \gamma_{L} \geqslant 0$ and $\gamma_{R}+\gamma_{L}<0$, from Lemma 1(d) we have $\Psi^{\prime \prime}(y ; b)<0$, and so system (5) has at most two crossing periodic orbits and from $(22)$ we get $\delta_{\infty}<0$. Then different cases arise. 
(i) If $0<b \ll 1$, then from Theorem 1 (a) we know that $\Psi(y ; 0) \neq 0$, so by continuity $\Psi(y ; b) \neq 0$ for $0<b \ll 1$ and $y \geqslant \hat{y}$, and the system has no crossing periodic orbits.

(ii) If $b \geqslant b_{C C}$, then from Lemma 2 , there exists a value $y_{R} \geqslant \hat{y}$ such that $\Psi\left(y_{R} ; b\right)>0$. Taking into account that $\Psi^{\prime \prime}(y ; b)<0$ and $\delta_{\infty}<0$, a elementary computation shows the existence of only one point $\bar{y}$ with $\Psi(\bar{y} ; b)=0$. Furthermore, since $\Psi^{\prime}(\bar{y} ; b)<0$, the point $\bar{y}$ corresponds to a stable crossing limit cycle.

(iii) If $0<b_{C C}-b \ll 1$, from Proposition 2 and Remark 4 we conclude the existence of two limit cycles, one unstable limit cycle for $y=$ $\hat{y}-\varepsilon_{1}$ and another one stable for $y=\bar{y}-\varepsilon_{2}$, being $\Psi\left(\bar{y} ; b_{C C}\right)=0$ and $\varepsilon_{1}>0, \varepsilon_{2}>0$ small enough. Hence by continuity there exists a value $b_{S N}<b_{C C}$ with only one crossing periodic orbit which is semi-stable and the statement is shown.

The proof of Theorem 3 follows.

Proof of Theorem 3: (a) When $\gamma_{R}+\gamma_{L} \geqslant 0$, then from Theorem 1(b) we have $\Psi(y ; 0) \neq 0$. Since $\Psi(0 ; 0)=-P_{L}(0)>0$, from Lemma $1(\mathrm{c})$ we get $\Psi(y ; b)>0$ in its domain, and there are no crossing periodic orbits.

(b) When $\gamma_{R}+\gamma_{L}<0$, then from Proposition 5 we have $\lim _{y \rightarrow \infty} P^{\prime}(y)<1$. Since $P(0)>0$, by using adequately the mean value theorem, we deduce that a point $\bar{y}$ exists with $P(\bar{y})=\bar{y}$, and the system has a crossing periodic orbit. Next we will prove the uniqueness of the fixed point $\bar{y}$. Assume on the contrary that Poincaré map $P$ has at least the two fixed points $b<\bar{y}_{1}<\bar{y}_{2}$ where $\bar{y}_{1}$ is the smallest fixed point and $\bar{y}_{2}$ is the consecutive one, then $P^{\prime}\left(\bar{y}_{1}\right) \leqslant 1$ and $P^{\prime}\left(\bar{y}_{2}\right) \geqslant 1$. From Proposition $5(\mathrm{~d})$ we obtain the derivative of Poincaré map at the fixed points $\bar{y}_{i}, i=1,2$ as follows,

$$
P^{\prime}\left(\bar{y}_{i}\right)=\frac{\bar{y}_{i}}{P_{L}\left(\bar{y}_{i}\right)} \frac{P_{L}\left(\bar{y}_{i}\right)-b}{\bar{y}_{i}-b} e^{2 \nu\left(\bar{y}_{i}\right)} \text {, where } \nu\left(\bar{y}_{i}\right)=\gamma_{L} t_{L i}+\gamma_{R} t_{R i} .
$$

Now, it is easy to see that

$$
\frac{\bar{y}_{1}}{\bar{y}_{1}-b}>\frac{\bar{y}_{2}}{\bar{y}_{2}-b}, \quad \frac{P_{L}\left(\bar{y}_{1}\right)-b}{P_{L}\left(\bar{y}_{1}\right)}>\frac{P_{L}\left(\bar{y}_{2}\right)-b}{P_{L}\left(\bar{y}_{2}\right)}, \quad \nu\left(\bar{y}_{1}\right)>\nu\left(\bar{y}_{2}\right)
$$

and so $P^{\prime}\left(\bar{y}_{2}\right)<P^{\prime}\left(\bar{y}_{1}\right) \leqslant 1$, which leads to a contradiction, and the system has only one crossing periodic orbit which is stable.

Before giving the proof of Theorems 4 and 5 , note that the asymptotes $A_{L}$ and $A_{R^{-1}}$, see Propositions 3 and 4 , intersect to the bisector 
of the first quadrant at the points $\left(2 x_{L} \gamma_{L}, 2 x_{L} \gamma_{L}\right)$ and $\left(b+2 x_{R} \gamma_{R}, b+\right.$ $\left.2 x_{R} \gamma_{R}\right)$ respectively.

Proof of Theorem 4: (a) When $b=b_{C C}$, according to Remark 5 we get $\Psi\left(\hat{y}+\varepsilon ; b_{C C}\right)>0$ for $\varepsilon>0$ small enough, and when $b>b_{C C}$, from Lemma 2 there exists $y_{R}>b$ such that $P_{R}^{-1}\left(y_{R} ; b\right)=0$, that is $\Psi\left(y_{R} ; b\right)=$ $-P_{L}\left(y_{R}\right)>0$. In both cases, $\delta_{\infty}<0$, see $(22)$, and from the intermediate value theorem a stable limit cycle exists.

(b) The asymptote $A_{L}$, see Proposition 3 , intersects to the bisector of the first quadrant at the point $\left(2 x_{L} \gamma_{L}, 2 x_{L} \gamma_{L}\right)$ satisfying $\left(A_{L}\right)^{\prime}(y)=-e^{\gamma_{L} \pi}$ and $A_{L}(y)<P_{L}(y)$ for $y \geqslant \hat{y}$. If $\gamma_{R}+\gamma_{L} \leqslant 0$, from Proposition 4(b) we get $-1<\left(P_{R}^{-1}\right)^{\prime}(y)<-e^{-\gamma_{R} \pi} \leqslant-e^{\gamma_{L} \pi}$. Since $P_{R}^{-1}(b)=b$, for $b<2 x_{L} \gamma_{L}$ we have $P_{R}^{-1}(y)<A_{L}(y)<P_{L}(y)$ and there are no crossing periodic orbits.

(c) If $b<b_{C C}$, then $\Psi(\hat{y} ; b)=P_{R}^{-1}(\hat{y})<0$. When $\gamma_{R}+\gamma_{L}>0$, from $(22)$ we get $\Psi(y ; b)>0$ for $y$ big enough, then the system has at least an unstable crossing periodic orbit.

(d) Since $\gamma_{L}<0$, from Proposition 3 we obtain

$$
P_{L}(y)=P_{L}(y)-P_{L}(\hat{y})=P_{L}^{\prime}(\xi)(y-\hat{y})<-e^{\gamma_{L} \pi}(y-\hat{y})=L(y),
$$

being $L(y)$ a straight parallel to the line $A_{L}(y)$, the asymptote of left Poincaré map $P_{L}$. From Proposition 4(c), the intersection $y_{A S R}$ of the asymptote $A_{R^{-1}}$ of the right Poincaré map with the $y$-axis is given by

$$
y_{A S R}=\left(1+e^{\gamma_{R} \pi}\right)\left(b+2 x_{R} \gamma_{R}\right) .
$$

Since $\gamma_{L}+\gamma_{R} \geqslant 0$ then the slope of the asymptote $A_{R^{-1}}$ is greater than the slope of the asymptote $A_{L}$ and for

$$
b>M=\frac{\hat{y}}{1+e^{\gamma_{R} \pi}}-2 x_{R} \gamma_{R}
$$

we get $y_{A S R}>\hat{y}$, that is $A_{R^{-1}}(y)>L(y)$. Thus, $y>\hat{y}$ we have

$$
P_{R}^{-1}(y ; b)>A_{R^{-1}}(y)>L(y)>P_{L}(y) .
$$

Hence $\Psi(y ; b)>0$ and there are no crossing limit cycles.

(e) Assume $\gamma_{R}=-\gamma_{L}$ and $b<b_{\infty}$, then $b+2 x_{R} \gamma_{R}<2 x_{L} \gamma_{L}$ and so, the two asymptotes $A_{R^{-1}}$ and $A_{L}$ are parallel and satisfy $A_{R^{-1}}(y)<A_{L}(y)$. Then there exists $y_{1}$ big enough such that $P_{R}^{-1}\left(y_{1}\right)<P_{L}\left(y_{1}\right)$, that is $\Psi\left(y_{1} ; b, \gamma_{R}\right)<0$. Take now $\gamma_{R}=-\gamma_{L}+\varepsilon$ with $\varepsilon>0$ enough small, then by continuity $\Psi\left(y_{1} ; b, \gamma_{R}+\varepsilon\right)<0$ and due to $\delta_{\infty}>0$, see $(22)$, there exists $y_{2}>y_{1}$ satisfying $\Psi\left(y_{2} ; b, \gamma_{R}+\varepsilon\right)>0$. Hence there exists $\bar{y}$ 
with $y_{2}>\bar{y}>y_{1}$ satisfying $\Psi\left(\bar{y} ; b, \gamma_{R}+\varepsilon\right)=0$ which corresponds to a unstable crossing periodic orbit.

The statement for $b>b_{\infty}$ is similar and it will not be shown.

Proof of Theorem 5: From Theorem 4(a), there is at least one stable crossing periodic orbit $\Gamma_{1}$ for $b=b_{C C}$, besides the crossing critical cycle. When $0<b_{C C}-b \ll 1$, from Proposition 2 an unstable limit cycle bifurcates from the crossing critical cycle and additionally, according to Remark 4, the stable crossing periodic orbit $\Gamma_{1}$ persists. Hence there are at least two crossing periodic orbits and statement (a) is shown.

To show statement (b) we must distinguish two cases.

(i) If $\gamma_{R}+\gamma_{L}=0$, the two asymptotes $A_{L}$ and $A_{R^{-1}}$ are parallel. For $b=b_{C C}<\hat{y}$, we get $b_{C C}+2 x_{R} \gamma_{R}<2 x_{L} \gamma_{L}$, so that $A_{R^{-1}}(y)<A_{L}(y)$. Then there exists a value $M>0$ such that for $y>M$ we have

$$
A_{R^{-1}}(y)<P_{R}^{-1}\left(y ; b_{C C}\right)<A_{L}(y)<P_{L}(y),
$$

that is $\Psi\left(y ; b_{C C}\right)<0$ for $y$ sufficiently big, while from Remark 5 we have $\Psi\left(\hat{y}+\varepsilon_{0} ; b_{C C}\right)>0$ for a certain $\varepsilon_{0}>0$. Thus a point $\bar{y} \neq \hat{y}$ with $\Psi\left(\bar{y} ; b_{C C}\right)=0$ and $\Psi^{\prime}\left(\bar{y} ; b_{C C}\right)<0$ exists and the system has a stable crossing periodic orbit, see Figure 9 . When $0<b-b_{C C} \ll 1$, from Proposition 2 and Remark 4 we conclude the existence of two limit cycles with opposite stabilities.

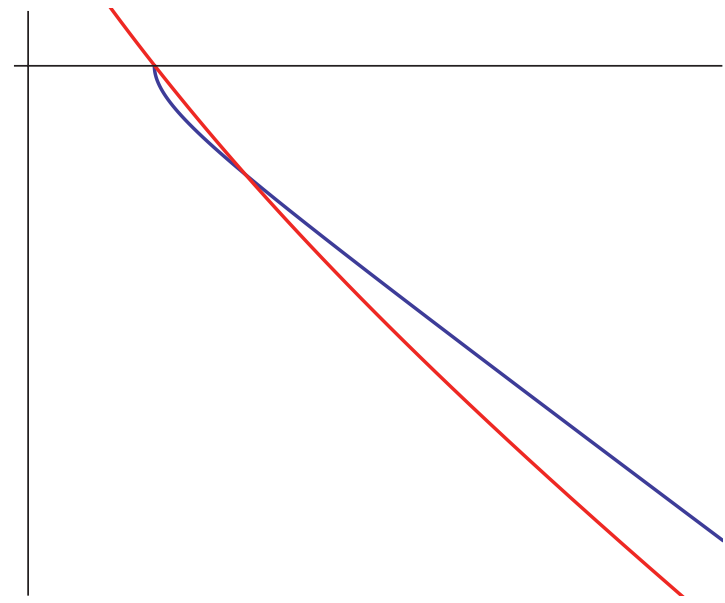

Figure 9. The Poincaré maps $P_{L}$ (blue) and $P_{R}^{-1}$ (red), for $0<-\gamma_{L}=\gamma_{R}$ and $b=b_{C C}\left(\gamma_{R}\right)$, showing the existence of one crossing limit cycle and the crossing critical cycle. 
(ii) Take $\gamma_{R}=-\gamma_{L}+\varepsilon_{2}$ with $\varepsilon_{2}>0$ and $b=b_{C C}\left(\gamma_{R}\right)$, see again Proposition 2. Now, according to Remark 4, the stable limit cycle existing for $\gamma_{R}=-\gamma_{L}$ persists, that is there exists a point $y_{1}$ with $P\left(y_{1}\right)=y_{1}$ and $P^{\prime}\left(y_{1}\right)<1$. Since $\lim _{y \rightarrow \infty} P^{\prime}(y)=e^{\left(\gamma_{R}+\gamma_{L}\right) \pi}>1$, a trivial analysis of the Poincaré map shows that there exists other point $y_{2}>y_{1}$ with $P\left(y_{2}\right)=y_{2}$ and $P^{\prime}\left(y_{2}\right)>1$ which corresponds to a unstable limit cycle, see Figure 10.

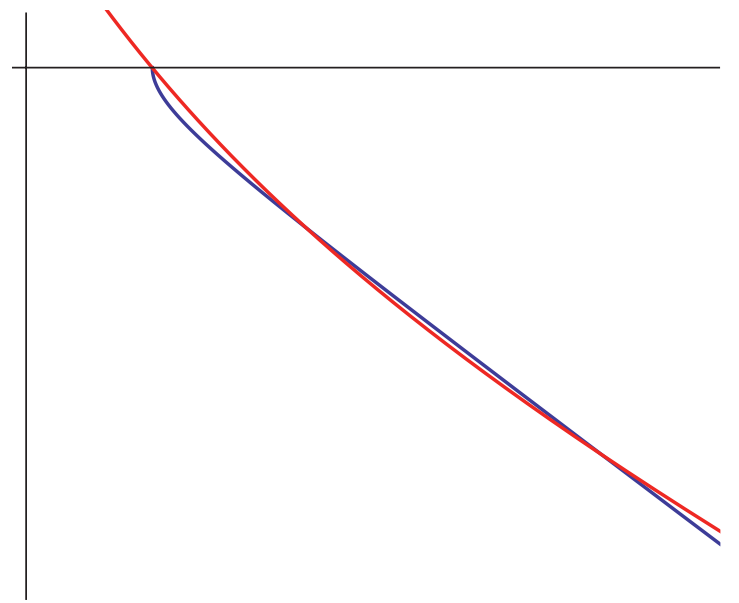

Figure 10. The Poincaré maps $P_{L}$ (blue) and $P_{R}^{-1}$ (red), for $0<-\gamma_{L}<\gamma_{R}<-\gamma_{L}+\varepsilon_{2}$ and $b=b_{C C}\left(\gamma_{R}\right)$, showing the existence of two crossing limit cycles and the crossing critical cycle.

Take now $\gamma_{R}=-\gamma_{L}+\varepsilon_{2}$ and $b=b_{C C}\left(\gamma_{R}\right)$ as before. If we perturb the parameter $b$ putting $b=b_{C C}-\varepsilon_{3}$, then according to Remark 4 the two crossing periodic orbits persist and according to Proposition 2 one unstable limit cycle bifurcates from the crossing critical cycle. Hence we have three crossing periodic orbits, see Figure 11, and we are done. 


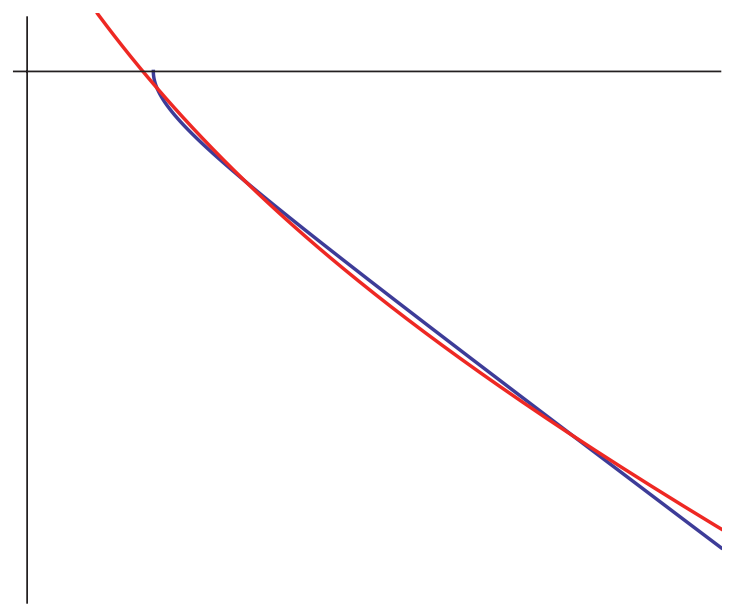

Figure 11. The Poincaré maps $P_{L}$ (blue) and $P_{R}^{-1}$ (red), for $0<-\gamma_{L}<\gamma_{R}<-\gamma_{L}+\varepsilon_{2}$ and $b=$ $b_{C C}\left(\gamma_{R}\right)-\varepsilon_{3}$, showing the existence of three crossing limit cycles.

\section{References}

[1] A. A. Andronov, A. A. Vitt, and S. E. Khaikin, "Theory of oscillators", Translated from the Russian by F. Immirzi; translation edited and abridged by W. Fishwick Pergamon Press, Oxford-New York-Toronto, Ont., 1966.

[2] D. De C. Braga And L. F. Mello, Limit cycles in a family of discontinuous piecewise linear differential systems with two zones in the plane, Nonlinear Dynam. (2013). DOI: 10.1007/s11071-013-0862-3.

[3] B. Coll, A. Gasull, and R. Prohens, Degenerate Hopf bifurcations in discontinuous planar systems, J. Math. Anal. Appl. 253(2) (2001), 671-690. DOI: 10.1006/jmaa.2000.7188.

[4] F. Dercole, S. Gragnani, and S. Rinaldi, Bifurcation analysis of piecewise smooth ecological models, Theoretical Population Biology 72(2) (2007), 197-213. DOI: 10.1016/j.tpb.2007.06.003.

[5] M. di Bernardo, C. J. Budd, A. R. Champneys, and P. KoWALCZYK, "Piecewise-smooth dynamical systems'. Theory and applications', Applied Mathematical Sciences 163, Springer-Verlag London, Ltd., London, 2008. 
[6] M. di Bernardo, M. I. Feigin, S. J. Hogan, And M. E. HoMER, Local analysis of $C$-bifurcations in $n$-dimensional piecewisesmooth dynamical systems, Chaos Solitons \& Fractals 10(11) (1999), 1881-1908. DOI : 10.1016/S0960-0779(98)00317-8.

[7] A. F. FilipPOV, "Differential equations with discontinuous righthand sides", Translated from the Russian, Mathematics and its Applications (Soviet Series) 18, Kluwer Academic Publishers Group, Dordrecht, 1988.

[8] E. Freire, E. Ponce, F. Rodrigo, and F. Torres, Bifurcation sets of continuous piecewise linear systems with two zones, Internat. J. Bifur. Chaos Appl. Sci. Engrg. 8(11) (1998), 2073-2097. DOI : $10.1142 / \mathrm{S} 0218127498001728$.

[9] E. Freire, E. Ponce, And F. Torres, Canonical discontinuous planar piecewise linear systems, SIAM J. Appl. Dyn. Syst. 11(1) (2012), 181-211. DOI: 10.1137/11083928X.

[10] F. Giannakopoulos and K. Pliete, Planar systems of piecewise linear differential equations with a line of discontinuity, Nonlinearity 14(6) (2001), 1611-1632. DOI: 10.1088/0951-7715/14/6/311.

[11] F. Giannakopoulos and K. Pliete, Closed trajectories in planar relay feedback systems, Special issue: Non-smooth dynamical systems, theory and applications, Dyn. Syst. 17(4) (2002), 343-358. DOI : $10.1080 / 1468936021000041681-1858$.

[12] M. Han And W. Zhang, On Hopf bifurcation in non-smooth planar systems, J. Differential Equations 248(9) (2010), 2399-2416. DOI : $10.1016 / j \cdot j$ de.2009.10.002.

[13] S.-M. HuAn And X.-S. Yang, On the number of limit cycles in general planar piecewise linear systems, Discrete Contin. Dyn. Syst. 32(6) (2012), 2147-2164. DOI: 10.3934/dcds.2012.32.2147.

[14] Yu. A. Kuznetsov, S. Rinaldi, and A. Gragnani, One-parameter bifurcations in planar Filippov systems, Internat. J. Bifur. Chaos Appl. Sci. Engrg. 13(8) (2003), 2157-2188. DOI : $10.1142 / \mathrm{S} 0218127403007874$.

[15] J. Llibre And E. Ponce, Three nested limit cycles in discontinuous piecewise linear differential systems with two zones, Dyn. Contin. Discrete Impuls. Syst. Ser. B Appl. Algorithms 19(3) (2012), 325-335.

[16] J. Llibre, E. Ponce, And F. Torres, On the existence and uniqueness of limit cycles in Liénard differential equations allowing discontinuities, Nonlinearity 21(9) (2008), 2121-2142. DOI : $10.1088 / 0951-7715 / 21 / 9 / 013$. 
[17] S. Shui, X. Zhang, And J. Li, The qualitative analysis of a class of planar Filippov systems, Nonlinear Anal. 73(5) (2010), 1277-1288. DOI: $10.1016 / j . n a .2010 .04 .053$.

[18] J. J. Stoker, "Nonlinear Vibrations in Mechanical and Electrical Systems", Interscience Publishers, Inc., New York, N.Y., 1950.

[19] Y. Zou, T. Küpper, AND W.-J. Beyn, Generalized Hopf bifurcation for planar Filippov systems continuous at the origin, J. Nonlinear Sci. 16(2) (2006), 159-177. DOI: 10.1007/s00332-005-0606-8.

E.T.S. Ingeniería

Universidad de Sevilla

Camino de los Descubrimientos

41092 Sevilla

Spain

E-mail address: efrem@us.es

E-mail address: eponcem@us.es

E-mail address: ftorres@us.es 